\title{
3D Epigenomic Characterization Reveals Insights Into Gene Regulation and Lineage Specification During Corticogenesis
}

Authors: Michael Song ${ }^{1,2, *}$, Mark-Phillip Pebworth ${ }^{3,4, *}$, Xiaoyu Yang ${ }^{1, *}$, Armen Abnousi $^{5}$, Changxu Fan ${ }^{6,7}$, Jia Wen ${ }^{8}$, Jonathan D. Rosen ${ }^{9}$, Mayank NK Choudhary ${ }^{6,7}$, Xiekui Cui ${ }^{1}$, Ian R. Jones ${ }^{1}$, Seth Bergenholtz ${ }^{1}$, Ugomma C. Eze ${ }^{4,9}$, Ivan Juric ${ }^{5}$, Bingkun $\mathrm{Li}^{1}$, Lenka Maliskova ${ }^{1}$, Weifang Liu ${ }^{9}$, Alex A. Pollen ${ }^{4,11}$, Yun $\mathrm{Li}^{8,9,12}$, Ting Wang ${ }^{6,7}$, Ming $\mathrm{Hu}^{5, *, \#,}$ Arnold R. Kriegstein ${ }^{4,11, \#, ~ Y i n ~ S h e n ~}{ }^{1,11, \#}$

\section{Affiliations}

${ }^{1}$ Institute for Human Genetics, University of California, San Francisco, San Francisco, CA, USA.

${ }^{2}$ Pharmaceutical Sciences and Pharmacogenomics Graduate Program, University of California, San Francisco, San Francisco, CA, USA.

${ }^{3}$ Biomedical Sciences Graduate Program, University of California, San Francisco, San Francisco, CA, USA.

${ }^{4}$ The Eli and Edythe Broad Center of Regeneration Medicine and Stem Cell Research, UCSF, San Francisco, CA, USA.

${ }^{5}$ Department of Quantitative Health Sciences, Lerner Research Institute, Cleveland Clinic Foundation, Cleveland, $\mathrm{OH}$, USA.

${ }^{6}$ Department of Genetics, Washington University School of Medicine, St. Louis, MO, USA. ${ }^{7}$ The Edison Family Center for Genome Sciences and Systems Biology, Washington University School of Medicine, St. Louis, MO, USA.

${ }^{8}$ Department of Genetics, University of North Carolina, Chapel Hill, NC, USA.

${ }^{9}$ Department of Biostatistics, University of North Carolina, Chapel Hill, NC, USA.

${ }^{10}$ Medical Scientist Training Program, University of California, San Francisco, San Francisco, CA, USA.

${ }^{11}$ Department of Neurology, University of California, San Francisco, San Francisco, CA, USA

${ }^{12}$ Department of Computer Science, University of North Carolina, Chapel Hill, NC, USA.

*These authors contributed equally to the work.

\#Corresponding authors: Ming Hu hum@ccf.org, Arnold R. Kriegstein Arnold.Kriegstein@ucsf.edu, and Yin Shen Yin.Shen@ucsf.edu. 
Abstract

Lineage-specific epigenomic changes during human corticogenesis have previously remained elusive due to challenges with tissue heterogeneity and sample availability. Here, we analyze cis-regulatory chromatin interactions, open chromatin regions, and transcriptomes for radial glia, intermediate progenitor cells, excitatory neurons, and interneurons isolated from mid-gestational human brain samples. We show that chromatin looping underlies transcriptional regulation for lineage-specific genes, with transcription factor motifs, families of transposable elements, and disease-associated variants enriched at distal interacting regions in a cell type-specific manner. A subset of promoters exhibit unusually high degrees of chromatin interactivity, which we term super interactive promoters. Super interactive promoters are enriched for critical lineagespecific genes, suggesting that interactions at these loci contribute to the fine-tuning of cell type-specific transcription. Finally, we present CRISPRview, a novel approach for validating distal interacting regions in primary cells. Our study presents the first characterization of cell type-specific 3D epigenomic landscapes during human corticogenesis, advancing our understanding of gene regulation and lineage specification during human brain development.

\section{Introduction}

The human cortex is a complex, heterogeneous structure that undergoes extensive expansion during development, a process which is markedly different and features distinct cell types from mouse cortical development. Previous studies utilized single cell RNA sequencing (scRNA-seq) to unravel the transcriptomic diversity of the developing cortex, revealing at least nine major cell types and up to 26 distinct subtypes in the dorsal

73 cortex alone ${ }^{1,2}$. Much of this diversity arises from cortical stem cells known as radial glia

74 (RG), whose cell bodies reside in the germinal zones (GZs) of the dorsal and ventral 75 cortex. In the dorsal cortex, RG divide asymmetrically to give rise to intermediate 76 progenitor cells (IPCs), which proliferate and differentiate into excitatory neurons (eNs) ${ }^{3,4}$. 77 These newborn neurons undergo radial migration until they reach the cortical plate (CP), 
78 where they mature and undergo synaptogenesis ${ }^{5}$. Meanwhile, interneurons (iNs)

79 produced in the ventral cortex migrate tangentially into the dorsal cortex through the marginal and germinal zones ${ }^{6}$. These processes result in a CP consisting primarily of eNs

81 and iNs and a GZ where all four cell types are intermixed.

Dynamic changes in the epigenomic landscape have been shown to play a critical role in development and cell fate commitment, for instance through the rewiring of physical chromatin loops between promoters and distal regulatory elements ${ }^{7}$. These regulatory interactions are of particular interest as their dysregulation has been linked to complex diseases and traits ${ }^{8,9}$. Despite their utility, detailed epigenomic characterizations are still absent for specific cell types in the developing cortex due to shortcomings associated with the analysis of bulk tissues ${ }^{10,11}$. Here, we present a novel strategy for isolating RG, IPCs, eNs, and iNs from mid-gestational human brain samples, enabling the cell typespecific profiling of their epigenomic features. In addition, we present CRISPRview, a technique for validating distal regulatory regions in primary cells, demonstrating that GPX3, TNC, and HES1 are regulated by distal enhancers in RG. Our results identify novel mechanisms underlying gene regulation and lineage specification during corticogenesis, providing a framework for the elucidation of diverse processes in development and disease.

\section{Results}

Isolation of specific cell populations from the developing human cortex.

To isolate four specific cell populations (RG, IPCs, eNs, and iNs) from mid-gestational 103 human brain samples between gestational weeks (GW) 15 to 22 (Supplementary Table

104 1), we adapted a previously reported approach for isolating RG from human cortical 105 samples using fluorescence-activated cell sorting (FACS) ${ }^{12}$. Specifically, we incorporated 106 markers for additional cell types from a recently published scRNA-seq dataset in the 107 human neocortex ${ }^{1}$. Microdissected GZ and CP samples were dissociated, stained using 108 antibodies for EOMES, SOX2, PAX6, and SATB2, then partitioned into their constituent 
109 populations using FACS (Fig. 1a and Supplementary Fig. 1). IPCs were first isolated as

110 the EOMES+ population. eNs were isolated from the EOMES- and SOX2- population

111 based on high expression of SATB2, which marks both newborn and mature eNs at the

112 ages of the samples ${ }^{1}$. RG were isolated based on the high expression of both SOX2 and

113 PAX6, and iNs were isolated based on medium SOX2 and low PAX6 expression.

114

115 The gene expression profiles of the sorted cell populations were both consistent with 116 cellular identity and reproducible between individuals (Fig. 1b and Supplementary Fig.

117 2a). Sorted RG expressed VIM, HES1, GPX3, and GFAP, with little to no expression of 118 marker genes for other cell types, whereas sorted IPCs expressed the IPC marker genes 119 EOMES, SSTR2, and NEUROD4. In concordance with previous reports, PAX6 was 120 expressed in both RG and IPCs. Sorted eNs expressed the eN marker genes SLA, 121 SLC17A7, SATB2, and TBR1, whereas $D L X 1, D L X 2$, and GAD1 were exclusively 122 detected in sorted iNs. When compared with aggregated scRNA-seq gene expression 123 profiles $^{1}$, our sorted cell populations exhibited the highest correlation with their 124 corresponding subtypes while also showing reduced correlation with cells from the 125 endothelial, mural, microglial, and choroid plexus lineages (Fig. 1c). Based on these 126 results, we determined that our sorting strategy was robust and our sorted cell populations 127 were suitable for additional epigenomic profiling.

129 Characterization of 3D epigenomic landscapes during corticogenesis.

131 We performed H3K4me3-centric proximity ligation-assisted ChIP-seq (PLAC-seq) to 132 identify chromatin interactions at active promoters, assay for transposase-accessible 133 chromatin using sequencing (ATAC-seq) to demarcate open chromatin regions, and RNA 134 sequencing (RNA-seq) to profile transcriptomes in the sorted RG, IPCs, eNs, and iNs 135 (Fig. 1d and Supplementary Table 2). We first confirmed the reproducibility of all PLAC136 seq and ATAC-seq replicates (Supplementary Fig. 2b and 2c). Next, we applied the 137 MAPS pipeline ${ }^{13}$ to call significant H3K4me3-mediated cis-regulatory chromatin 138 interactions in merged replicates for each cell type at a resolution of $5 \mathrm{~kb}$. We identified $13935,552,26,138,29,104$ and 22,598 MAPS interactions for RG, IPCs, eNs, and iNs, 
140 respectively, with approximately $85 \%$ of the interactions classified as anchor to non-

141 anchor (XOR), and the remaining interactions classified as anchor to anchor (AND) (Fig.

142 1e and Supplementary Fig. 3a and $\mathbf{3 b}$ ). The median interaction distance was between

$143170 \mathrm{~kb}$ to $230 \mathrm{~kb}$ for each cell type (Fig. 1f), and the majority of interactions occurred

144 within TADs in GZ and CP tissues ${ }^{10}$ (Supplementary Fig. 3c).

145

146

H3K4me3-mediated chromatin interactions contribute to cell type-specific gene expression.

Since $\mathrm{H} 3 \mathrm{~K} 4 \mathrm{me} 3$ is a histone mark associated with active promoters, we were interested to determine to what extent 3K4me3-mediated chromatin interactions influenced gene expression. First, we observed that the sorted cell populations clustered by developmental age based on their interaction strengths (Fig. 2a). This is consistent with iNs at this age possessing several characteristics of progenitor cells such as high SOX2 expression (Fig. 1a and 1b). Genes participating in cell type-specific interactions are enriched for biological processes associated with their respective cell types, including cell proliferation for RG and IPCs, neuron projection development for IPCs and eNs, and synaptogenesis for eNs (Supplementary Fig. 4a and Supplementary Table 3). In addition, based on comparing interaction strength and gene expression side-by-side, we observe the two to be generally correlated (Fig. 2a). In fact, interaction strength and gene expression are globally correlated across all pairwise comparisons of cell types (Fig. 2b and Supplementary Fig. 4b), suggesting that gene expression is orchestrated by physical chromatin looping in a manner that is highly cell type-specific.

To investigate how chromatin interactions contribute to gene regulation in greater detail, we took advantage of the enrichment of open chromatin regions at distal interacting regions (Fig. 2c and Supplementary Fig. 4c) and performed transcription factor (TF) motif enrichment analysis using $\mathrm{HOMER}^{14}$ at the set of cell type-specific distal interacting regions for each cell type (Fig. 2d and Supplementary Table 4). PAX6, EOMES, and TBR1 are the most highly enriched motifs in RG, IPCs, and eNs, respectively, recapitulating their sequential expression along this developmental axis ${ }^{15}$. Meanwhile, 
171 motifs for progenitor-specific TFs including EMX1, EMX2, and LHX2 are enriched in RG

172 and IPCs. The motif for RFX4, which was previously identified as an RG marker in the 173 murine midbrain as well as the human telencephalon, is enriched in $\mathrm{RGs}^{1,16}$. Finally, the 174 DLX1, DLX2, DLX6, GSX2, and LHX6 motifs are enriched in iNs, consistent with their 175 roles in iN maturation and function ${ }^{17-19}$. Overall, our approach identifies both known and 176 novel associations between TF binding at distal interacting regions and processes linked 177 to cellular identity.

Super interactive promoters are enriched for lineage-specific genes.

The number of chromatin interactions at H3K4me3-marked promoters is only modestly correlated with gene expression (Supplementary Fig. 5a). One explanation is that

183 individual genes are expressed to varying degrees in the context of their diverse cellular 184 functions, and regulatory elements are better described as fine-tuning rather than independently inducing or silencing the expression of their cognate promoters. Multiple 186 regulatory interactions can also exert synergistic or nonlinear effects on gene expression. 187 To examine the relationship between gene expression and chromatin interactivity in 188 greater detail, we first demonstrate that cell type-specific genes tend to have more 189 interactions than shared genes across all cell types (Fig. 3a and Supplementary Fig. 190 5b). Next, by ranking promoter-containing anchor bins according to their cumulative 191 interaction scores, we identify a subset of promoters with unusually high degrees of 192 chromatin interactivity, which we term super interactive promoters (SIPS) (Fig. 3b). In 193 total, we annotate 755, 765, 638, and 663 SIPs in RG, IPCs, eNs, and iNs, respectively 194 (Fig. 3c and Supplementary Table 5). SIPs are enriched for key lineage-specific genes 195 including GFAP and HES1 for RG, EOMES for IPCs, SATB2 for eNs, and GAD1, GAD2, $196 D L X 5, D L X 6$, and $L H X 6$ for iNs. SIPs are also frequently shared across multiple cell types. 197 For example, we identify SIPs for FOXG1 and POU3F3 (BRN1) in all four cell types, 198 SOX2 in the progenitor-like RG, IPCs, and iNs, and TBR1 in the eN-like IPCs and eNs. 199 Interestingly, a large number of promoters for lincRNA genes including LINC00461 and 200 LINC01551 are annotated as SIPs, consistent with their patterns of expression in the 201 developing $\operatorname{cortex}^{20}$. Globally, SIPs are enriched for in cell types with the highest 
202 expression of their genes among all four cell types, supporting their putative roles in 203 lineage specification (Fig. 3d). To assess whether SIPs are a general feature for other 204 cell types, we expanded our analysis to hematopoietic lineages with published promoter 205 capture Hi-C datasets ${ }^{21}$. Consistent with our results in brain cells, SIPs identified in 206 neutrophils, naive CD4+ T cells, monocytes, megakaryocytes, and erythroblasts are also 207 enriched for cell type-specific over shared genes (Fig. 3e). Based on these lines of 208 evidence, SIPs may represent a general mechanism used by cells to maintain the precise 209 and robust expression of key genes underlying cellular identity and function.

Specific families of transposable elements are implicated in SIP formation.

213 Given the important roles SIPs may harbor in establishing cellular identity, we were 214 interested in exploring potential mechanisms underlying their formation and evolution.

215 Towards this goal, we evaluated the contribution of transposable elements (TEs), which 216 are capable of propagating regulatory elements across the genome and influencing 3D 217 genome architecture $22-24$. First, we analyzed the enrichment of TEs at the class, family, 218 and subfamily levels in sequences defined by the union of SIPs and their distal interacting 219 regions (SIP groups or SIPGs) (Fig. 3f and Supplementary Fig. 6a-c). Notably, the 220 ERVL-MaLR family and many of its subfamilies are enriched in SIPGs across all four cell 221 types. Since we detected the strongest enrichment of this family of TEs in eNs, we 222 decided to focus on this particular lineage. In total, we identified 16 SIPGs in eNs that are 223 statistically enriched for ERVL-MaLR TEs (hypergeometric test, $P<0.01$ ) (Fig. 3g). Next, 224 we used HOMER to perform TF motif enrichment analysis at ERVL-MaLR TEs within 225 these 16 SIPGs and determined ZNF143 to be the most enriched motif (Fig. 3h). ZNF143 226 is an architectural protein which has previously been reported to mediate looping between 227 promoters and distal regulatory elements ${ }^{25}$. Moreover, certain subfamilies of ERVL-MaLR 228 TEs have been demonstrated to contribute to ZNF143 binding in 3T3 and HeLa cells ${ }^{26}$. 229 The ADRA2A SIPG in eNs exhibited the highest enrichment of ERVL-MaLR-derived 230 ZNF143 motifs (hypergeometric test, $P=1.59 \times 10^{-6}$ ) (Fig. 3i) and was associated with 231 elevated ADRA2A expression in eNs (Supplementary Fig. 6d). It spans 42 distal 232 interacting regions, 25 of which contain ERVL-MaLR TEs, and 12 of which contain one 
233 or more ERVL-MaLR-derived ZNF143 motifs (Fig. 3j and Supplementary Fig. 6e).

234 Furthermore, ZNF143 motifs in TEs from multiple ERVL-MaLR subfamilies (THE1A,

235 THE1C, MSTA) within the SIPG can be mapped back to ZNF143 motifs in the consensus

236 sequences of the same subfamilies (Supplementary Fig. 6f). This supports a model in

237 which ZNF143 motifs are coordinately expanded by ERVL-MaLR TE insertion, leading to

238 increased binding site redundancy and strengthened assembly of the ADRA2A SIPG

239 regulatory unit (Fig. 3k). Our results imply that TEs are capable of mediating the formation

240 of higher order epigenomic features including SIPs ${ }^{27}$.

Investigating features of the developmental trajectory from RG to eNs during corticogenesis.

Since RG, IPCs, and eNs represent a developmental trajectory from dorsal cortex progenitors to mature functional neurons, we grouped genes according to their expression and cumulative interaction scores along this axis and identified groups corresponding to RG, IPCs, and eNs (groups 1-3) that are enriched for lineage-defining genes and biological processes (Fig. 4a, Supplementary Fig. 7, and Supplementary Table 6). We also identified groups with decreasing expression and increasing chromatin interactivity (group 4) as well as increasing expression and decreasing chromatin interactivity (group 5) from RG to eNs, which could represent late-silenced and early-

253 silenced genes, respectively. Late-silenced genes are enriched for chromatin remodeling and epigenetic regulation terms, whereas early-silenced genes are enriched for eNspecific signatures. These results demonstrate that gene expression can be mediated by distinct modes of chromatin-mediated regulation during development.

Human corticogenesis is dramatically distinct from that in other mammalian species, 259 driven in large part by the increased diversity and proliferative capacity of cortical progenitors during development which results in the increased size and complexity of the human brain ${ }^{28}$. In particular, Notch signaling genes have been implicated in the clonal 262 expansion of RG, which constitute the major subtype of cortical progenitors in the 263 cortex ${ }^{29,30}$. Here, we find that RG are enriched relative to other cell types for chromatin 
264 interactions at Notch signaling genes from the AmiGO database ${ }^{31}$ (Fig. 4b). Compared

265 to other cell types, chromatin interactions in RG also target a significantly higher

266 proportion of human gained enhancers identified through comparative analyses of

267 H3K4me2 and H3K27ac ChIP-seq signal in human, rhesus macaque, and mouse

268 brains $^{32}$. Therefore, 3D epigenomic landscapes are capable of identifying lineage-specific

269 pathways contributing to human-specific aspects of cortical development. In addition, we

270 provide detailed annotations of gene targets for human gained enhancers and in vivo-

271 validated enhancer elements from the Vista Enhancer Browser ${ }^{33}$ in Supplementary

272 Table 7.

273

274 Leveraging 3D epigenomic landscapes to partition heritability for complex neuropsychiatric disorder- and trait-associated variants.

Chromatin interactions identified in our sorted cell populations represent a unique in vivo resource for mapping complex neuropsychiatric disorder- and trait-associated variants to their target genes (Figure 4c and Supplementary Table 8). They additionally enable the assessment of cell type-specific patterns of SNP heritability. To partition SNP heritability using our 3D epigenomic annotations, we employed linkage disequilibrium score regression (LDSC) ${ }^{34,35}$ using summary statistics from genome wide association studies (GWAS) for the following neuropsychiatric traits: Alzheimer's disease (AD), attention deficit hyperactivity disorder $(A D H D)^{36}$, autism spectrum disorder $(A S D)^{37}$, bipolar disorder $(B D)^{38}$, intelligence quotient $(I Q)^{39}$, major depressive disorder $(M D D)^{40}$, and schizophrenia $(\mathrm{SCZ})^{41}$. Overall, we observed significant levels of heritability enrichment in 3D anchor bins for every cell type and neuropsychiatric trait we evaluated $(15.13<$ enrichment score < 51.57, 1.27x10-40 < $P$ < 0.02) (Fig. 4d). These findings are largely expected as the majority of interacting promoters are shared across our cell types. When we restricted our analysis to 3D target bins, we observed dramatically distinct patterns of

291 cell type-specific heritability enrichment (Fig. 4e). For example, ASD SNP heritability was 292 significantly enriched for only in RG and eNs $\left(P=1.07 \times 10^{-3}\right.$ and $4.75 \times 10^{-4}$, respectively), 293 and AD SNP heritability was significantly enriched for only in RG $\left(P=3.99 \times 10^{-6}\right)$. Our

294 findings reflect the cell type-specific nature of distal regulatory elements that are 
295 dysregulated during disease and underscore the importance of leveraging 3D epigenomic

296 annotations to interpret variants that are located in non-coding regions of the genome.

Functional characterization of enhancers in primary cells using CRISPRview.

Validating distal regulatory elements in primary cells has historically been challenging, with most experiments to date performed using cell lines or iPSC-derived cells ${ }^{10,42}$. Here, we present CRISPRview, a novel approach combining CRISPRi, RNAscope, and immunostaining to validate enhancers in heterogeneous cultures of primary cells at the single cell level (Fig. 5a). We use CRISPRview to validate multiple enhancers in RG at the GPX3, TNC, and HES1 loci, all of which harbor RG-specific chromatin interactions and are differentially expressed in RG (Fig. 5b-d). Furthermore, the promoters for TNC and HES1 are annotated as SIPs in RG. First, sgRNAs were designed to target open chromatin regions physically interacting with the promoters of GPX3, TNC, and HES1 (Supplementary Table 9). Next, primary cultures of microdissected and dissociated GZ samples between GW17 to GW19 were infected with lentivirus expressing the experimental sgRNA, dCas9-KRAB, and mCherry in combination with lentivirus expressing control sgRNA, dCas9-KRAB, and GFP. After five additional days in culture,

313 the cells were fixed and stained using antibodies for mCherry, GFP, the RG marker

314 GFAP, and RNAscope probes $\left(A C D^{T M}\right)$ targeting intronic regions of the genes of interest.

315 Finally, high resolution images were taken using confocal microscopy, and the number of

316 punctate dots representing individual nascent transcripts were compared between

317 experimental (mCherry+) and control (GFP+) sgRNA-treated GFAP+ RG (Fig. 5b-d). The

318 SMART-Q pipeline was specifically developed in our lab for image analysis (see attached 319 manuscript, submitted).

321 All four regions interacting with the GPX3 promoter (regions 1-4) were found to exhibit 322 downregulation of GPX3 expression upon CRISPRi targeting (Fig. 5b). Notably, region 1 323 overlaps both a human gained enhancer ${ }^{32}$ and a Vista enhancer element (mm1343) $)^{33}$, 324 supporting its function as an enhancer in RG. Next, we investigated the locus for TNC, a 325 RG-specific gene implicated in neuronal migration, axon guidance, and synaptic plasticity. 
326 We found that two of its interacting regions exhibited significant downregulation of TNC

327 expression upon CRISPRi targeting (regions 1 and 2), but that silencing of its other two 328 interacting regions did not result in notable changes in TNC expression (regions 3 and 4)

329 (Fig. 5c). This could be due to the presence of alternative regulatory elements or 330 structural interactions at these loci. Finally, HES1 is a lineage-defining TF for RG, and we 331 positively validated all three regions that were found to interact with the HES1 promoter 332 (regions 1-3) (Fig. 5d). The observation of small but significant changes in gene 333 expression upon CRISPRi targeting supports the hypothesis that multiple interactions 334 work in concert to titrate the expression of key genes linked to cellular identity. In addition, 335 the observed broad distributions of nascent transcript counts likely reflects the stochastic 336 nature of transcription in single cells, stressing the importance of employing an approach 337 combining resolution, sensitivity, and cell type-specificity for validating enhancers in 338 single cells.

\section{Discussion}

342 Recent publications leveraging single cell sequencing have highlighted the heterogeneity of the developing human cortex, underscoring the necessity of studying epigenomic regulation in a cell type-specific manner. Within the dorsal cortex alone, there are a massive variety of cell types from multiple sources both within and outside the developing neural tube, including RG, IPCs, eNs, MGE-derived iNs, CGE-derived iNs, microglia, endothelial cells, and subplate neurons. Despite large differences in maturation state and

348 lineage, many of these cell types share intriguing similarities in terms of gene expression.

349 For example, iNs express a number of TFs typically associated with RG proliferation such 350 as SOX2 or eN differentiation such as ASCL1 and NPAS3 ${ }^{1}$. Therefore, bulk 351 measurements cannot reliably reveal the nuanced epigenomic programs driving gene 352 expression in each cell type. By profiling 3D epigenomic landscapes in specific cell 353 populations during corticogenesis, we not only demonstrate that gene regulation is closely 354 linked to chromatin interactivity, we also identify SIPs that are highly cell type-specific and 355 enriched for key lineage-specific genes. We uncover a potential mechanism by which 356 specific families of TEs propagate binding sites for architectural proteins, facilitating the 
357 formation of multi-interaction clusters which may serve to sustain gene expression. While

358 the analysis of TEs is currently constrained by the list of known motifs and the resolution

359 of chromatin interactions identified in this study, future advances will help us further

360 elucidate the contribution of TEs to 3D genome architecture and transcriptional regulation.

362 Cortical progenitors, eNs, and iNs are highly divergent in terms of their diversity, 363 proliferative capacity, distribution, and functional characteristics between humans and 364 mice ${ }^{28}$. Therefore, processes occurring during human cortex development cannot be fully recapitulated with mouse models. These non-murine features also indicate that enhancer mutations in humans may not adequately phenocopy to mice. Our dataset provides a comprehensive catalog of annotations for human gained enhancers and complex neuropsychiatric disorder- or trait-associated variants in cell types that are intricately tied to human cortex development, enabling the interpretation and prioritization of regulatory sequences for follow-up studies.

372 Finally, there is a need to perform cell type-specific validation for regulatory sequences in 373 primary cells, especially as our understanding of epigenomic regulation matures over

374 time. By combining immunostaining for cellular markers with the quantification of nascent 375 transcripts in the nucleus, CRISPRview offers exquisite sensitivity and resolution for 376 detecting cell type-specific changes in gene expression in single cells. Here, we used 377 CRISPRview to successfully validate multiple regulatory elements in RG and observed 378 subtle but significant changes in gene expression at the GPX3, TNC, and HES1 loci. 379 Further experiments leveraging CRISPRview in live tissue cultures should continue to 380 reveal novel regulatory logic in a manner that is truly representative of the complex in vivo 381 environment that is present during human cortex development.

\section{Acknowledgements}

This work was supported by the UCSF Weill Institute for Neuroscience Innovation Award 386 (to Y.S. and A.R.K.), the National Institutes of Health (NIH) grants R01AG057497, 387 R01EY027789 and UM1HG009402 (to Y.S.), R35NS097305 (to A.R.K.), the Hillblom 
388 Foundation, and the American Federation for Aging Research New Investigator Award in

389 Alzheimer's Disease (to Y.S). This work was also supported by NIH grants 390 R01HL129132, U544HD079124, and R01MH106611 (to Y.L.), R01HG007175, 391 U24ES026699, and U01HG009391 (to T.W.), and the American Cancer Society grant 392 RSG-14-049-01-DMC (to T.W). M.S. is supported by T32GM007175. M.P. is supported 393 by the National Science Foundation Graduate Research Fellowship grant number 394 1650113. U.C.E. is supported by 5T32GM007618-42. This work was made possible in 395 part by NIH grants P30EY002162 to the UCSF Core Grant for Vision Research, 396 P30DK063720, and S101S10OD021822-01 to the UCSF Parnassus Flow Cytometry 397 Core.

\section{Author contributions}

Y.S., A.A.P., and A.R.K. conceived the study. Y.S., M.H., A.A.P., A.R.K. supervised the study. M.S., M.P., X.Y., I.R.J., X.C., U.C.E., and L.M. performed experiments. M.S., A.A., S.B., J.D.R., B.L., I.J., and M.H. performed computational analysis. C.F. and M.N.C. performed transposable element analysis under the supervision of T.W. J.W. and W.L. performed SNP heritability analysis under the supervision of Y.L. M.S., M.P., X.Y., and Y.S. analyzed and interpreted the data. Y.S., M.S., M.P., X.Y., and M.H prepared the manuscript with input from all other authors.

Competing interests statement

411 The authors declare no competing financial interests.

\section{Code availability statement}

415 A copy of the custom code used for data analysis and figure generation in this study is 416 available upon request. 
420 All datasets used in this study (PLAC-seq, ATAC-seq, RNA-seq) are available at the

421 Neuroscience Multi-Omic Archive (NeMO Archive) under controlled access. Chromatin

422 interactions, open chromatin regions, and gene expression results for each cell type can

423 be accessed from the NeMO Archive using the following link:

424 https://assets.nemoarchive.org/dat-uioqy8b

426 Data can also be visualized on the WashU Epigenome Browser using the following link:

427 http://epigenomegateway.wustl.edu/legacy/?genome=hg38\&session=0Czw03b5/z\&stat

$428 \underline{\text { usld }=1958712809}$

Figure legends

Figure 1. Experimental design and general features of the 3D epigenomic landscape during corticogenesis.

(a) Schematic of the sorting strategy. Within the dorsal cortex, the germinal zone (GZ) is populated by radial glia (RG), which extend fibers towards the cortical plate (CP). These $R G$ divide asymmetrically to produce intermediate progenitor cells (IPCs), which differentiate into excitatory neurons (eNs) that migrate along $R G$ fibers towards the CP. At the same time, interneurons (iNs) can be found in both the $\mathrm{GZ}$ and $\mathrm{CP}$. Microdissected $G Z$ and $C P$ samples were dissociated into single cells before being fixed, stained with antibodies for EOMES, SOX2, PAX6, and SATB2, and sorted using FACS. (b) Heatmap showing expression of key marker genes for RG, IPCs, eNs, and iNs. (c) Heatmap showing correlations between gene expression profiles for sorted cell populations and

444 aggregate gene expression profiles from scRNA-seq datasets in the developing cortex.

445 Cell types include newborn iNs from the medial ganglionic eminence (MGE), ventral 446 progenitors including RG and IPCs from the MGE, microglia, and choroid plexus cells. (d) 447 WashU Epigenome Browser snapshot of a $360 \mathrm{~kb}$ region (chr17:72,970,0000$44873,330,000)$ showing IPC-specific chromatin interactions linked to SSTR2 expression in IPCs. (e) Bar graph showing counts of MAPS interactions, with proportions of XOR (blue, 
450 only one interacting bin contains H3K4me3 peaks) and AND (red, both interacting bins

451 contain H3K4me3 peaks) interactions displayed for each cell type. (f) Cumulative

452 distribution function (CDF) plots showing interaction distances for each cell type. (g)

453 Histogram showing the numbers of MAPS interactions at each promoter for each cell 454 type.

Figure 2. H3K4me3-mediated chromatin interactions contribute to cell type-specific gene regulation.

(a) Heatmaps displaying interaction scores (left) and gene expression (right) for unique

XOR interactions grouped according to their cell type specificity. Hierarchical clustering dendrograms for each heatmap are also shown (top). (b) Scatterplot showing positive correlation between the difference in the number of MAPS interactions at each promoter and the difference in expression of the corresponding genes between RG and eNs (Pearson product-moment correlation coefficient, two-sided t-test, $P<2.2 \times 10^{-16}$ ). The fitted trendline based on linear regression is also shown. (c) Fold enrichment of open chromatin regions over distance-matched background regions in $1 \mathrm{Mb}$ windows around distal interacting regions for MAPS interactions in RG. (d) Enrichment of TF motifs at open chromatin regions in cell type-specific interacting distal regions for each cell type. The color of each dot represents the degree of enrichment (- $\log _{10} \mathrm{P}$-value) for each TF motif, and the size of each dot represents the gene expression of the corresponding TF.

Figure 3. Super interactive promoters are enriched for lineage-specific genes.

(a) CDF plots showing the numbers of MAPS interactions for shared versus cell typespecific genes in eNs (two sample t-test, two-sided, $P=1.40 \times 10^{-14}$ ). (b) Plots showing 476 the ranked cumulative interaction scores for 3D anchor bins in each cell type, defined as 477 the sum of the $-\log _{10}$ FDR for MAPS interactions coincident to each bin. Super interactive 478 promoters (SIPs) are defined as promoters located to the right of the knee of each curve 479 (dashed lines). Example SIPs, including those for lineage-specific genes, are highlighted 480 for each cell type. (c) Venn diagram displaying the cell type-specificity of SIPs in RG, 
481 IPCs, eNs, and iNs. (d) The number of genes called as SIPs was divided by the total 482 number of SIPs and non-SIPs for genes with the $1^{\text {st }}, 2^{\text {nd }}, 3^{\text {rd }}$, and $4^{\text {th }}$ highest expression 483 among all four cell types. The fold enrichment was calculated relative to the group with 484 the $4^{\text {th }}$ highest expression for each cell type. (e) Forrest plot showing that SIPs called in 485 hematopoietic cells are also enriched for cell type-specific over shared genes. 95\% 486 confidence intervals are displayed. (f) Scatterplot showing both the enrichment and the 487 number of observed copies of TE families in SIPGs for eNs. TE families occupying more 488 than $1 \%$ of the genome are colored. (g) Scatterplot showing the enrichment of ERVL489 MaLR TEs in SIPGs for eNs (hypergeometric P-value, see methods). SIPGs with 40 or 490 more distal interacting bins and $P<0.01$ are highlighted. (h) Scatterplot showing the 491 enrichment of TF motifs at ERVL-MaLR TEs in SIPGs highlighted in (g). Only TF motifs 492 with length > $12 \mathrm{bp}$ are shown. (i) Scatterplot showing the enrichment of ZNF143 motifs 493 at ERVL-MaLR TEs in SIPGs highlighted in (g) (Poisson distribution, see methods). 494 ZNF143 motifs occurrences were detected using FIMO using a threshold of $P=0.0001$.

495 (j) WashU Epigenome Browser snapshot of the ADRA2A SIPG. MAPS interactions 496 targeting the 12 distal interacting bins containing ERVL-MaLR-derived ZNF143 motifs are 497 highlighted. (k) Potential mechanism for the contributions of TEs towards SIP formation.

Figure 4. Investigating developmental trajectories during corticogenesis and partitioning heritability for complex neuropsychiatric disorders and traits.

(a) Gene groups identified based on their changes in expression and chromatin interactivity along the transition from RG to eNs. Group 1 represents stem cell genes with decreasing expression and chromatin interactivity from RG to eNs. Group 2 represents IPC-specific genes with the highest expression and chromatin interactivity at the IPC stage. Group 3 represents genes with increasing expression and chromatin interactivity from RG to eNs. Groups 4 and 5 are characterized by anti-correlated expression and chromatin interactivity and may represent late-silenced and early-silenced genes, respectively. Representative genes and GO terms are shown for each group. (b) Bar

510 graph showing the numbers of MAPS interactions at Notch signaling genes targeting bins 511 with and without human gained enhancers in each cell type (Chi-square test). (c) Bar 
512 graph showing the numbers of unique GWAS SNPs $\left(P<10^{-8}\right)$ interacting with their

513 nearest gene only, with both their nearest and distal genes, or with distal genes only for

514 each cell type across all neuropsychiatric traits. (d-e) LDSC enrichment scores for each

515 neuropsychiatric trait and cell type, stratified by 3D anchor and target bins. Results with

$516 P>0.05$ are indicated.

Figure 5. Functional characterization of distal interacting regions using 519 CRISPRview.

(a) CRISPRview workflow. Image analysis was performed using the SMART-Q pipeline.

(b-d) Functional characterization of distal interacting regions at the GPX3, TNC, and interaction bridging the promoters of GPX3, TNC, and HES1 and distal interacting regions containing open chromatin regions (highlighted) which were targeted by sgRNAs for CRISPRi silencing. Representative images show staining for RNAscope probes targeting 527 intronic regions for the genes of interest (white), DAPI (blue), the RG marker GFAP (light 528 blue), mCherry (red), and GFP (green). The scale bar is $50 \mu \mathrm{m}$. Box plots show the results 529 of CRISPRi silencing for each targeted region. The open circles represent single cells, 530 and nascent transcript counts for experimental (mCherry+) versus control (GFP+) 531 sgRNA-treated RG are represented on the y-axis (Student's t-test, two-tailed). The 532 median, upper and lower quartiles, and $10 \%$ to $90 \%$ range are indicated.

Supplemental Figure 1. Representative contour plots depicting FACS gating strategy.

(a) Cells were separated from debris of various sizes based on the forward scatter area 538 (FSC-A) and side scatter area (SSC-A). Cells were then passed through two singlet gates 539 using the width and height metrics of the (b) side scatter (SSC-H versus SSC-W) and (c) 540 forward scatter (FSC-H versus FSC-W). (d) SOX2+, and SOX2-, and intermediate 541 progenitor (IPC) populations were isolated by gating on EOMES-PE-Cy7 and SOX2542 PerCP-Cy5.5 staining. (e) Radial glia (RG) and interneurons (iNs) were isolated as high 
543 PAX6/high SOX2 and medium SOX2/low PAX6 populations, respectively. (f) Excitatory 544 neurons (eNs) were isolated from the SOX2- population by gating on SATB2-Alexa Fluor 545647 staining.

Supplementary Figure 2. Reproducibility between replicates for RNA-seq, ATACseq, and PLAC-seq.

(a) RNA-seq replicates were hierarchically clustered according to gene expression sample distances using DESeq2. (b) Heatmap with pairwise correlations and hierarchical clustering for read densities at the set of unified open chromatin regions for ATAC-seq replicates. (c) Principle component analysis (PCA) was performed based on the normalized contact frequencies across all PLAC-seq replicates (see methods). To assess the robustness of the results, we conducted the analysis separately for bin pairs within 300 and $600 \mathrm{~kb}$ interacting windows.

\section{Supplementary Figure 3. Identification of chromatin interactions using MAPS.}

(a) Illustration of AND and XOR sets in a representative PLAC-seq contact matrix. The cells represent interactions within the same bin. The purple cells represent interactions in

563 the AND set where both of the interacting bins contain 1D H3K4me3 peaks. The orange 564 cells represent interactions in the XOR set where only one of the interacting bins contains 565 1D H3K4me3 peaks. The grey cells represent interactions where neither of the interacting 566 bins contains 1D H3K4me3 peaks. (b) Venn diagram displaying cell type-specificity of

567 MAPS interactions for each cell type. (c) Proportions of MAPS interactions occurring 568 within and across TADs in GZ and CP tissues for each cell type. regulation. 
573 (a) GO enrichment analysis for genes whose promoters participate in cell type-specific 574 interactions. The top annotation clusters from DAVID are reported along with their 575 enrichment scores for each cell type. (b) Scatterplots showing positive correlation 576 between the difference in the number of MAPS interactions at each promoter and the 577 difference in expression of the corresponding genes between all pairs of cell types 578 (Pearson product-moment correlation coefficient, two-sided t-test, $P<2.2 \times 10^{-16}$ for all cell 579 types). Fitted trendlines based on linear regression are also shown. (c) Fold enrichment 580 of open chromatin regions over distance-matched background regions in $1 \mathrm{Mb}$ windows 581 around distal interacting regions for MAPS interactions in IPCs, eNs, and iNs.

\section{Supplementary Figure 5. Correlations between chromatin interactions and gene} expression for cell-type specific and shared genes.

(a) Scatterplots showing the correlation between numbers of MAPS interactions and gene expression at promoters in each cell type. (b) Cumulative distribution function (CDF) plots showing the numbers of MAPS interactions for shared versus cell type-specific genes in

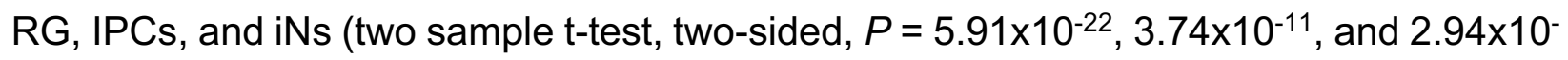
19 for RG, IPCs, and iNs, respectively).

Supplementary Figure 6. Specific families of transposable elements are implicated in SIP formation.

(a-c) Enrichment of TEs in SIPGs at the class (a), family (b), and subfamily (c) levels for each cell type. Only families occupying more than $1 \%$ of the genome are shown in (b). 597 Only subfamilies from the MIR and ERVL-MaLR families occupying more than $0.1 \%$ of 598 the genome are shown in (c). (d) Bar graph shows elevated ADRA2A gene expression in 599 eNs. (e) Illustration of the 12 distal interacting regions in the ADRA2A SIPG containing at 600 least one ERVL-MaLR-derived ZNF143 motif. ZNF143 motifs are indicated and colored 601 by strand. The bin identifier corresponds to the labels in Fig. 3j. (f) Illustration of the 602 conservation of ZNF143 binding motifs in ERVL-MaLR TEs. Blue bars indicate consensus 603 sequences, yellow bars indicate individual copies of ERVL-MaLR TEs in the ADRA2A 
604 SIPG, and red bars indicate ZNF143 motifs. The positions of the ZNF143 motifs relative

605 to the ERVL-MaLR TE sequences was determined using FIMO.

606

607 Supplementary Figure 7. Developmental trajectories and annotations for complex 608 neuropsychiatric disorder- and trait-associated variants.

610 (a) Box plots showing the distributions of gene expression and cumulative interaction 611 scores for groups in Fig. 4. The median, upper and lower quartiles, minimum, and 612 maximum are indicated. (b) Bar graphs showing the numbers of GWAS SNPs $\left(P<10^{-8}\right)$ 613 interacting with their nearest gene only, with both their nearest and distal genes, or with 614 distal genes only for each cell type and neuropsychiatric trait. Venn diagrams display the 615 cell type-specificity of all interacting GWAS SNPs for each neuropsychiatric trait.

617 Supplementary Table 1. Sample metadata.

619 Supplementary Table 2. PLAC-seq, ATAC-seq, and RNA-seq data processing 620 metrics.

622 Supplementary Table 3. Enriched GO terms for genes participating in cell type623 specific interactions. regions.

Supplementary Table 5. Super interactive promoters for each cell type.

Supplementary Table 6. Enriched GO terms for genes associated with specific developmental trajectories. 
636 Supplementary Table 8. Target gene annotation for complex neuropsychiatric disorder- and trait-associated GWAS SNPs overlapping chromatin interactions.

Supplementary Table 9. sgRNA sequences used for functional validation.

\section{Methods}

642

\section{Ethics statement}

Deidentified embryonic brain tissue samples were collected with prior patient consent in

647 by the Human Gamete, Embryo, and Stem Cell Research Committee (GESCR) and 648 Institutional Review Board at the University of California, San Francisco.

\section{Tissue dissociation}

652 The tissue dissociation protocol was adapted from Nowakowski et al, 2017 ${ }^{1}$. Briefly,

653 samples were cut into small pieces in artificial cerebrospinal fluid before being added into 654 pre-warmed papain dissociation media (Worthington \#LK003150). The dissociation 655 solution was incubated for 45 minutes at $37^{\circ} \mathrm{C}$. Excess solution was removed and 656 replaced with cell culture media. The pieces of tissue were triturated, filtered through a $65770 \mu \mathrm{M}$ nylon mesh, and centrifuged at $300 \mathrm{~g}$ for 8 minutes. The supernatant was removed 658 and replaced with fresh culture media.

\section{Sample fixation}

662 Mid-gestational human brain samples between GW15 and GW22 were fixed in 2\% 663 paraformaldehyde prepared in PBS with gentle agitation for 10 minutes at room 664 temperature. Glycine was added to a final concentration of $200 \mathrm{mM}$ to quench the 
665 reactions, and the samples were centrifuged at $500 \mathrm{~g}$ for 5 minutes at $4^{\circ} \mathrm{C}$. The samples

666 were washed twice with PBS before being frozen at $-80^{\circ} \mathrm{C}$ until further processing.

\section{Permeabilization and staining}

The cell pellet was thawed on ice and resuspended in PBS with 0.1\% Triton-X-100 for 15

671 minutes. The cells were then washed twice with PBS and resuspended in 5\% BSA in PBS 672 for staining. Staining proceeded for at least one hour with FcR Blocking Reagent (Miltenyi 673 Biotech, 1/20 dilution), EOMES-PE-Cy7 (Invitrogen, WD1928, 1/10 dilution), PAX6-PE 674 (BD Biosciences, O18-1330, 1/10 dilution), SOX2-PerCP-Cy5.5 (BD Biosciences, O38675 678, 1/10 dilution), and SATB2-Alexa Fluor 647 (Abcam, EPNCIR130A, 1/100 dilution).

676 Following staining, the cells were centrifuged at $500 \mathrm{~g}$ for 5 minutes. The supernatant was 677 removed, and the pellet was diluted into PBS. When sorting cells for RNA-seq libraries, 678 1\% RNasin Plus RNase Inhibitor (Promega) was included in all buffers, PBS was 679 prepared from RNase-free stocks, and acetylated RNase-free BSA was used to prepare $6805 \%$ BSA in PBS for staining.

\section{FACS}

AbC Total Antibody Compensation Beads (Thermo Fisher) were used to generate single color compensation controls prior to sorting. Sorting was conducted on either the FACSAria II, FACSAria Ilu, or FACSAria Fusion instruments using a $70 \mu \mathrm{M}$ nozzle, and

687 cells were collected in $5 \mathrm{ml}$ tubes pre-coated with FBS. A sample of each sorted population was reanalyzed on the same machine to assess purity. The cells were collected by centrifuging at $500 \mathrm{~g}$ for 10 minutes. The supernatant was removed, and the

690 pellet was frozen at $-80^{\circ} \mathrm{C}$ until further processing. When sorting cells for RNA-seq

691 libraries, collection tubes were coated with both FBS and RNAlater (Thermo Fisher)

\section{Primary cell culture}

695 Following dissociation, cells were plated onto Matrigel-coated coverslips in 48 well plates 
696 at a density of approximately $0.7 \times 10^{6}$ cells per well. The cells were infected with lentivirus

69724 hours after plating, and media was changed every two days. Media was composed of

698 96\% DMEM/F-12 with GlutaMAX, 1\% N-2, 1\% B-27, and 1\% penicillin/streptomycin. The

699 cells were grown in $8 \%$ oxygen and 5\% carbon dioxide, and they were harvested for

700 fixation four days post-infection.

701

702

\section{PLAC-seq}

703

704 PLAC-seq was performed according to the protocol from Fang et al., $2016^{43}$. 1 to 5 million 705 cells were used to prepare each library. Digestion was performed using $100 \mathrm{U}$ Mbol for 2 706 hours at $37^{\circ} \mathrm{C}$, and chromatin immunoprecipitation was performed using Dynabeads M707280 sheep anti-rabbit IgG (Invitrogen \#11203D) mixed with 5 Mg anti-H3K4me3 antibody 708 (Millipore 04-745). TruSeq sequencing adapters were added during PCR amplification. 709 Libraries were sent for paired-end sequencing on the HiSeq X Ten or NovoSeq 6000 710 instruments (150 bp paired-end reads). fastp was applied to trim reads to $100 \mathrm{bp}$, and 711 replicates were merged and downsampled to normalize the number of usable reads 712 before processing with MAPS.

MAPS interaction calling

715

716 We used the MAPS pipeline to call significant long-range chromatin interactions from our 717 PLAC-seq datasets. First, bwa mem was used to map raw reads to hg38. Unmapped 718 reads and reads with low mapping quality were discarded, and the resulting filtered read 719 pairs were processed as previously reported ${ }^{13}$. Briefly, we divided the genome into $5 \mathrm{~kb}$ 720 bins and counted the number of read pairs representing interactions between $5 \mathrm{~kb}$ bins. 721 To define our 3D anchor bins, we took the union of peaks called using MACS2 from read 722 pairs with interaction distances < $1 \mathrm{~kb}$ for each cell type (1D H3K4me3 peaks). Based on 723 this annotation, we classified interactions into AND, XOR, and NOT sets based on 724 whether both, only one, or none of the interacting $5 \mathrm{~kb}$ bins overlapped 1D H3K4me3 725 peaks (Supplementary Fig. 3a). Since we were interested in identifying significant 726 H3K4me3-mediated chromatin interactions, we retained only interactions in the AND and 
727 XOR sets for downstream processing. We also retained only intrachromosomal

728 interactions with interaction distances between $10 \mathrm{~kb}$ and $1 \mathrm{Mb}$. These two criteria

729 constituted our definition of usable reads.

731 For calling interactions, we applied a Poisson regression-based approach to normalize

732 systematic biases from restriction sites, GC content, sequence repetitiveness, and ChIP

733 enrichment. We fitted models for interactions in the AND and XOR sets separately and

734 calculated false discovery rates (FDRs) for interactions based on their expected and

735 observed contact frequencies between $5 \mathrm{~kb}$ bin pairs. Furthermore, we grouped

736 interactions that were located within $15 \mathrm{~kb}$ of each other at both ends into clusters and

737 classified all other interactions as singletons. To define our sets of significant long-range

738 chromatin interactions, we retained only interactions with 12 or more reads, normalized

739 contact frequencies (defined as the ratio between the observed and expected contact

740 frequencies) $\geq 2$, and FDR $<0.01$ for clusters and FDR $<0.0001$ for singletons. This was

741 based on the reasoning that more biologically meaningful interactions are likely to appear

742 in clusters, and singletons are more likely to present false positives.

\section{MAPS reproducibility analysis}

746 PCA plots were generated based on the normalized contact frequencies for $5 \mathrm{~kb}$ bin pairs

747 from our PLAC-seq datasets. Specifically, we first extracted AND and XOR 5 kb bin pairs

748 based on cell type-specific 1D H3K4me3 peaks for each of the 11 replicates. We next

749 applied zero-truncated Poisson regression, adjusting for the same systematic biases as

750 the MAPS pipeline. Again, we derived normalized contact frequencies based on the ratio

751 between the observed and expected contact frequencies between $5 \mathrm{~kb}$ bin pairs, with the

752 expected contact frequencies being the fitted values from the zero-truncated Poisson

753 regression. Normalized contact frequencies were then log-transformed and merged

754 across the 11 replicates. This quantile normalized merged data was used to generate the

755 PCA plots. We restricted our analysis to $5 \mathrm{~kb}$ bin pairs within $300 \mathrm{~kb}$ or $600 \mathrm{~kb}$ windows

756 for Supplementary Fig. 2c. 
ATAC-seq was performed as previously described using the Nextera DNA Library Prep Kit (Illumina \#FC-121-1030). First, fixed cells were washed once with ice cold PBS containing $1 \mathrm{x}$ protease inhibitor before being resuspended in ice cold nuclei extraction buffer (10 mM Tris-HCl pH 7.5, $10 \mathrm{mM} \mathrm{NaCl}, 3 \mathrm{mM} \mathrm{MgCl} 2,0.1 \%$ Igepal CA630, and 1x protease inhibitor) for 5 minutes. Next, 50,000 cells were counted out, exchanged into 50 $\mu \mathrm{L} 1 \mathrm{x}$ Buffer TD, then incubated with $2.5 \mu \mathrm{L}$ TDE1 enzyme for 45 minutes at $37^{\circ} \mathrm{C}$ with shaking. Following transposition, $150 \mu \mathrm{L}$ reverse crosslinking solution $(50 \mu \mathrm{L} 1 \mathrm{M}$ Tris $\mathrm{pH}$ 8.0, $100 \mu \mathrm{L}$ 10\% SDS, $2 \mu \mathrm{L} 0.5 \mathrm{M}$ EDTA, $10 \mu \mathrm{L} 5 \mathrm{M} \mathrm{NaCl}, 800 \mu \mathrm{L}$ water, and $2.5 \mu \mathrm{L} 20$ $\mathrm{mg} / \mathrm{mL}$ Proteinase $\mathrm{K}$ ) was added to each reaction, and the reactions were incubated at $65^{\circ} \mathrm{C}$ overnight. On the next day, DNA was purified using Qiagen MinElute spin columns, amplified using Nextera primers, then size-selected for fragments between 300 and 1000 bp using AMPure XP beads. Libraries were sent for paired-end sequencing on the NovaSeq 6000 instrument (150 bp paired-end reads). Raw reads were mapped to hg38 and processed using

the ENCODE pipeline (https://github.com/kundajelab/atac_dnase_pipelines) running the default settings. All sequencing reads were trimmed to $50 \mathrm{bp}$ prior to mapping. The sets of optimal naive overlap peaks for each cell type were used for further downstream analysis.

\section{RNA-seq}

779

We extracted total RNA from the sorted cell populations using the RNAstorm ${ }^{\text {TM }}$ FFPE RNA extraction kit (Cell Data Sciences \#CD501) starting from $5 \times 10^{5}$ to $1.5 \times 10^{6}$ cells. The quality of the extracted RNA was checked by calculating the percentage of RNA 783 fragments with size > $200 \mathrm{bp}$ (DV200) from the Agilent 2100 Bioanalyzer. RNA samples 784 with DV200 >= 40\% were used for library construction. First, they were depleted of 785 ribosomal RNA using the KAPA RNA HyperPrep Kit with RiboErase (HMR \#KK8560).

786 Next, the RNA was used for first and second strand synthesis, dA-tailing, and sequencing 787 adapter ligation. The cDNA was cleaned up and TruSeq sequencing adapters were added 
788 via PCR amplification. Libraries were sent for paired-end sequencing on the NovaSeq 7896000 instrument (150 bp paired-end reads). Raw reads were aligned to hg38 using STAR 790 running the standard ENCODE parameters, and transcript quantification was performed 791 in a strand-specific manner using RSEM with the GENCODE 29 annotation. The edgeR 792 package in R was used to calculate TMM-normalized RPKM values for each gene based 793 on the expected counts and gene lengths for each replicate as reported by RSEM. The 794 mean gene expression across all replicates for each cell type was used for further 795 downstream analysis.

796

\section{TF motif enrichment analysis}

799 We took the set of open chromatin regions participating in cell type-specific XOR 800 interactions for each cell type and used the sequences in $200 \mathrm{bp}$ windows around the 801 peak summits to perform motif enrichment analysis using HOMER running the default 802 settings. The complete set of vertebrate motifs from the JASPAR database were used for 803 detection. The "-float" option was specified to optimize the detection threshold, and the 804 entire genome was used as a background. Entries with similar or identical consensus TF motif sequences were grouped for brevity.

806

\section{GO enrichment analysis}

808

809 Protein coding and noncoding RNA genes from GENCODE 29 participating in cell type810 specific XOR interactions were used for GO enrichment analysis. Only genes participating 811 in interactions with promoter open chromatin regions on one end and distal open

812 chromatin regions on the other end were used. A minimum normalized RPKM of 0.5 was 813 used to filter out genes that were not significantly expressed the corresponding cell types, 814 and the resulting gene lists were input into DAVID 6.8 running functional annotation 815 clustering with the default settings and the "GOTERM_BP_ALL" ontology.

\section{SIP identification}


819 We devised an approach similar to calling super-enhancers ${ }^{44}$ to identify super interactive 820 promoters (SIPs) using our MAPS interactions for each cell type. Specifically, we started

821 from 18,373 anchor bins containing 1D H3K4me3 peaks annotated in at least one of the 822 sorted cell populations. For each anchor bin, we calculated the cumulative interaction 823 score for all its coincident MAPS interactions. For anchor bins without any MAPS 824 interactions, the cumulative interaction score was calculated to be zero. We then 825 prepared plots of the ranked cumulative interaction scores for anchor bins in each cell 826 type and defined SIPs as promoters located to the right of the knee of each curve.

\section{Defining cell type-specific versus shared genes}

We classified each gene as cell type-specific or shared according to its Shannon entropy 831 across all four cell types. Specifically, we first calculated the relative expression of each 832 gene in each cell type, defined as a gene's normalized RPKM in the cell type divided by 833 the sum of the gene's normalized RPKMs across all four cell types. Next, we calculated 834 the Shannon entropy based the gene's relative expression in each of the cell types. A cell 835 type-specific gene is characterized by low entropy, while a shared gene is characterized 836 by high entropy. We classified a gene as cell type-specific if met the following conditions:

837 its entropy was < 0.01 , its normalized RPKM was $>1$ in that cell type, and its normalized 838 RPKM was highest in that cell type among all four cell types. All other genes with 839 normalized RPKM > 1 across every cell type were classified as shared.

TE family and subfamily enrichment in SIPGs

843 A SIP and its distal interacting regions are considered to be a SIP group or SIPG. TE 844 enrichment in SIPGs was evaluated as follows. The foreground enrichment was defined 845 as the number of copies of TEs from a given family or subfamily overlapping SIPGs in 846 each cell type. The background enrichment was defined as the number of copies of TEs 847 overlapping all interacting $5 \mathrm{~kb}$ bins. The overall enrichment was defined as the 848 foreground enrichment divided by the background enrichment multiplied by the fraction 
849 of interacting $5 \mathrm{~kb}$ bins belonging to SIPGs. At least $50 \%$ of a TE had to intersect a $5 \mathrm{~kb}$

850 bin for it to be considered to overlap the $5 \mathrm{~kb}$ bin.

ERVL-MaLR TEs enrichment in specific SIPGs

854 For each SIPG, the foreground enrichment was defined as the number of distal interacting 855 regions with one or more copies of an ERVL-MaLR TE. The background enrichment was 856 calculated by randomly permuting the locations of the distal interacting regions and 857 counting the number of permuted regions with one or more copies of an ERVL-MaLR TE. 858 This was performed over 100 such permutations. The overall enrichment was defined as 859 the foreground enrichment divided by the background enrichment. The one-tailed P-value 860 for each SIPG was calculated using the hypergeometric distribution as follows: $P=$ 861 choose $(m, q) \times$ choose $(n, k-q) /$ choose $(m+n, k)$, where " $q$ " is the number of $5 k b$ bins 862 within the SIPG with one or more copies of an ERVL-MaLR TE, " $m$ " is the number of $5 \mathrm{~kb}$ 863 bins with one or more copies of an ERVL-MaLR TE on the same chromosome, " $n$ " is the 864 number of $5 \mathrm{~kb}$ bins with no copies of an ERVL-MaLR TE on the same chromosome, and 865 " $k$ " is the size of the SIPG.

ZNF143 motif enrichment at ERVL-MaLR TEs in specific SIPGs

869 For each SIPG, the foreground enrichment was defined as the number of ZNF143 motifs occurring in ERVL-MaLR TEs as determined using FIMO in distal interacting regions for

871 the SIPG. The background enrichment was defined as the number of ZNF143 motifs 872 occurring in the SIPG, but not necessarily in the ERVL-MaLR-TEs. The overall

873 enrichment was defined as the foreground enrichment divided by the background 874 enrichment multiplied by fraction of the SIPG sequence occupied by ERVL-MaLR TEs. 875 The one-tailed P-value was calculated using the Poisson distribution as follows: the 876 number of events is the foreground enrichment, and the probability is the background 877 enrichment multiplied by fraction of the SIPG sequence occupied by ERVL-MaLR TEs. 
Target gene annotation for enhancers and complex neuropsychiatric disorder- and trait-associated variants

To determine whether a human gained enhancer, Vista enhancer element, or GWAS SNP 883 potentially interacted with a target gene, we determined whether any of its promoters 884 participated in MAPS interactions with the feature of interest on the other end. All human 885 gained enhancers and Vista enhancer elements were expanded to a width of $5 \mathrm{~kb}$ and all 886 GWAS SNPs were expanded to a width of $1 \mathrm{~kb}$ to account for potential functional sequences around each feature. Furthermore, we determined the proportion of GWAS SNPs interacting with their nearest and/or distal genes, except when the promoters for the nearest gene and GWAS SNP fell within the same $5 \mathrm{~kb}$ bin and could not be resolved for MAPS interactions ("same fragment ambiguity"). We provide target gene annotations for human gained enhancers and Vista enhancer elements in Supplementary Table 7 and GWAS SNPS in Supplementary Table 8. The overlap of each feature with open chromatin regions in each cell type is also reported.

Partitioning heritability for complex neuropsychiatric disorder- and trait-

We employed stratified LD score regression ${ }^{34,35}$ to partition SNP heritability for neuropsychiatric traits using our cell type-specific 3D epigenomic annotations. Specifically, we first collected GWAS summary statistics for seven complex neuropsychiatric disorders and traits including Alzheimer's disease (AD), attention deficit hyperactivity disorder (ADHD), autism spectrum disorder (ASD), bipolar disorder (BD),

903 intelligence quotient (IQ), major depressive disorder (MDD), and schizophrenia 904 (SCZ). We estimated the enrichment of SNP heritability for each complex 905 neuropsychiatric disorder and trait separately based on 3D anchor or target bins from 906 MAPS interactions for each cell type. 3D anchor bins contain H3K4me3 ChIP-seq peaks 907 and are presumably enriched for active promoters, whereas 3D target bins are 908 presumably enriched for distal regulatory elements such as enhancers. 


\section{Validation of distal interacting regions using CRISPRview}

912 The CRISPRi vector was modified from the Mosaic-seq ${ }^{45}$ and CROP-seq vectors ${ }^{46}$. The

913 hU6-sgRNA expression cassette from the CROPseq-Guide-Puro vector (Addgene

914 \#86708) was cloned and inserted downstream of the WPRE element in the Lenti-dCas9-

915 KRAB-blast vector (Addgene \#89567). The blasticidin resistance gene was replaced with

916 either mCherry or EGFP. sgRNAs targeting open chromatin regions in distal interaction

917 regions were designed using CHOPCHOP. Single stranded DNA was annealed and

918 ligated into the CRISPRi vector at the BsmBI cutting locus. Single clones were picked

919 following transformation, and the sgRNA sequences were confirmed by Sanger

920 sequencing. For lentiviral packaging, the CRISPRi vector, pMD2.G (Addgene \#12259),

921 and psPAX (Addgene \#12260) were transformed into 293T cells using PolyJet (SignaGen

922 Laboratories \#SL100688) according to the manufacturer's instructions. Virus-containing

923 media was collected three times every 16 to 20 hours and concentrated using Amicon

$92410 \mathrm{~K}$ columns. Collected lentivirus was stored immediately at $-80^{\circ} \mathrm{C}$. Primary cell cultures

925 were infected with virus (MOI < 1) 24 hours after plating, and four days after infection,

926 cells were harvested and fixed with 4\% PFA for FISH and immunostaining.

928 FISH experiments detecting nascent transcripts were performed using the RNAScope 929 Multiplex Fluorescent V2 Assay kit (ACDBio \#323100) followed by immunostaining for 930 cell type-specific markers. Probes targeting intronic regions for GPX3 (ACDBio \#572341), 931 TNC (ACDBio \#572361), and HES1 (ACDBio \#560881) were custom-designed, 932 synthesized, and labeled with TSA Cyanine 5 (Perkin Elmer \#NEL705A001KT, 1:1000 933 dilution). Next, fixed cells were pretreated with hydrogen peroxide for 10 minutes and 934 Protease III for 15 minutes, and probes were hybridized and amplified according to the 935 manufacturer's instructions. Slides were washed with PBS before blocking with 5\% 936 donkey serum in PBS for 30 minutes at room temperature. Next, slides were incubated 937 with primary antibodies against mCherry (Abcam ab205402), GFP (Abcam ab1218) and 938 GFAP (Abcam ab7260) overnight at $4^{\circ} \mathrm{C}$, followed by incubation with Alexa Fluor 488 939 donkey anti-mouse IgG (Thermo Fisher Scientific \#A21202), Alexa-546 nm donkey anti940 rabbit IgG (Thermo Fisher Scientific \#A10040), or Alexa-594 nm goat anti-chicken IgG 
941 (Thermo Fisher Scientific \#A11042) for 1 hour at room temperature. Three-dimensional 942 confocal microscopy images were captured using a Leica TCS SP8 with a 40x oil943 immersion objective lens (NA = 1.30). The z-step size was $0.4 \mu \mathrm{m}$. For five color 944 multiplexed imaging, three sequential scans were performed to avoid overlapping 945 spectra. The first excitation lasers were $405 \mathrm{~nm}$ and $594 \mathrm{~nm}$, the second excitation lasers 946 were $488 \mathrm{~nm}$ and $633 \mathrm{~nm}$, and the third excitation laser was $561 \mathrm{~nm}$. All images were 947 obtained using the same acquisition settings. For FISH analysis, we developed an 948 integrated Python-based pipeline called Single-Molecule Automatic RNA Transcription 949 Quantification (SMART-Q) for quantifying nascent transcripts in single cells. Briefly, 950 RNAscope signal was filtered then fitted in three dimensions using Gaussian models.

951 Next, segmentation was performed on the DAPI channel in two dimensions to ascertain 952 the location of each nucleus. Finally, segmentation was performed on the cell marker 953 channel to identify RG-specific nuclei, and the positional RNAscope data was integrated 954 with the segmentation results to determine the final quantification of nascent transcripts 955 in each cell.

\section{References}

9591 Nowakowski, T. J. et al. Spatiotemporal gene expression trajectories reveal developmental 960 hierarchies of the human cortex. Science 358, 1318-1323, doi:10.1126/science.aap8809 961 (2017).

9622 Zhong, S. et al. A single-cell RNA-seq survey of the developmental landscape of the human prefrontal cortex. Nature 555, 524-528, doi:10.1038/nature25980 (2018).

9643 Hansen, D. V., Lui, J. H., Parker, P. R. \& Kriegstein, A. R. Neurogenic radial glia in the outer subventricular zone of human neocortex. Nature 464, 554-561, doi:10.1038/nature08845 (2010).

4 Pontious, A., Kowalczyk, T., Englund, C. \& Hevner, R. F. Role of intermediate progenitor cells in cerebral cortex development. Dev Neurosci 30, 24-32, doi:10.1159/000109848 (2008). $\mathrm{Wu}$, Q. et al. The dynamics of neuronal migration. Adv Exp Med Biol 800, 25-36, 5 Wu, Q. et al. The dynamics of neuronat

6 Anderson, S., Mione, M., Yun, K. \& Rubenstein, J. L. Differential origins of neocortical projection and local circuit neurons: role of Dlx genes in neocortical interneuronogenesis. Cereb Cortex 9, 646-654, doi:10.1093/cercor/9.6.646 (1999).

7 Zheng, H. \& Xie, W. The role of 3D genome organization in development and cell

9788 Li, Y., Hu, M. \& Shen, Y. Gene regulation in the 3D genome. Hum Mol Genet 27, R228differentiation. Nat Rev Mol Cell Biol 20, 535-550, doi:10.1038/s41580-019-0132-4 (2019).

R233, doi:10.1093/hmg/ddy164 (2018). 
9809 Schoenfelder, S. \& Fraser, P. Long-range enhancer-promoter contacts in gene expression 981 control. Nat Rev Genet 20, 437-455, doi:10.1038/s41576-019-0128-0 (2019).

98210 Won, $\mathrm{H}$. et al. Chromosome conformation elucidates regulatory relationships in

983

984 developing human brain. Nature 538, 523-527, doi:10.1038/nature19847 (2016).

985

11 Roadmap Epigenomics, C. et al. Integrative analysis of 111 reference human epigenomes. Nature 518, 317-330, doi:10.1038/nature14248 (2015).

98612 Thomsen, E. R. et al. Fixed single-cell transcriptomic characterization of human radial glial diversity. Nat Methods 13, 87-93, doi:10.1038/nmeth.3629 (2016).

13 Juric, I. et al. MAPS: Model-based analysis of long-range chromatin interactions from PLAC-seq and HiChIP experiments. PLoS Comput Biol 15, e1006982, doi:10.1371/journal.pcbi.1006982 (2019).

99114 Heinz, S. et al. Simple combinations of lineage-determining transcription factors prime cis-regulatory elements required for macrophage and B cell identities. Mol Cell 38, 576589, doi:10.1016/j.molcel.2010.05.004 (2010).

99415 Englund, C. et al. Pax6, Tbr2, and Tbr1 are expressed sequentially by radial glia, intermediate progenitor cells, and postmitotic neurons in developing neocortex. J Neurosci 25, 247-251, doi:10.1523/JNEUROSCI.2899-04.2005 (2005).

16 La Manno, G. et al. Molecular Diversity of Midbrain Development in Mouse, Human, and Stem Cells. Cell 167, 566-580 e519, doi:10.1016/j.cell.2016.09.027 (2016).

17 Neves, G. et al. The LIM homeodomain protein Lhx6 regulates maturation of interneurons

1001

1002 and network excitability in the mammalian cortex. Cereb Cortex 23, 1811-1823, doi:10.1093/cercor/bhs159 (2013).

1003

18 Wang, B. et al. Loss of Gsx1 and Gsx2 function rescues distinct phenotypes in Dlx1/2 mutants. J Comp Neurol 521, 1561-1584, doi:10.1002/cne.23242 (2013).

1004

19 Wang, Y. et al. Dlx5 and Dlx6 regulate the development of parvalbumin-expressing

1005 cortical interneurons. J Neurosci 30, 5334-5345, doi:10.1523/JNEUROSCI.5963-09.2010

1006

1007 (2010).

1008

20 Liu, S. J. et al. Single-cell analysis of long non-coding RNAs in the developing human neocortex. Genome Biol 17, 67, doi:10.1186/s13059-016-0932-1 (2016).

1009

21 Javierre, B. M. et al. Lineage-Specific Genome Architecture Links Enhancers and Non-

1010

1011

1012 coding Disease Variants to Target Gene Promoters. Cell 167, 1369-1384 e1319, doi:10.1016/j.cell.2016.09.037 (2016).

1013

22 Feschotte, C. Transposable elements and the evolution of regulatory networks. Nat Rev Genet 9, 397-405, doi:10.1038/nrg2337 (2008).

1014

23 Zhang, Y. et al. Transcriptionally active HERV-H retrotransposons demarcate topologically associating domains in human pluripotent stem cells. Nat Genet, doi:10.1038/s41588-019-0479-7 (2019).

1016

1017

24 Choudhary, M. N. K. et al. Co-opted transposons help perpetuate conserved higher-order chromosomal structures. bioRxiv, doi:https://doi.org/10.1101/485342 (2018). at gene promoters. Nat Commun 2, 6186, doi:10.1038/ncomms7186 (2015). Nucleic Acids Res 41, 4000-4014, doi:10.1093/nar/gkt088 (2013). 
102427 Sundaram, V. \& Wang, T. Transposable Element Mediated Innovation in Gene Regulatory

1025

1026

1027

1028

1029

1030

1031

1032

1033

1034

1035

1036

1037

1038

1039

1040

1041

1042

1043

1044

1045

1046

1047

1048

1049

1050

1051

1052

1053

1054

1055

1056

1057

1058

1059

1060

1061

1062

1063

1064

1065

1066

1067

1068

1069

Landscapes of Cells: Re-Visiting the "Gene-Battery" Model. Bioessays 40, doi:10.1002/bies.201700155 (2018).

28 Miller, D. J., Bhaduri, A., Sestan, N. \& Kriegstein, A. Shared and derived features of cellular diversity in the human cerebral cortex. Curr Opin Neurobiol 56, 117-124, doi:10.1016/j.conb.2018.12.005 (2019).

29 Suzuki, I. K. et al. Human-Specific NOTCH2NL Genes Expand Cortical Neurogenesis through Delta/Notch Regulation. Cell 173, 1370-1384 e1316, doi:10.1016/j.cell.2018.03.067 (2018).

30 Rani, N. et al. A Primate lncRNA Mediates Notch Signaling during Neuronal Development by Sequestering miRNA. Neuron 90, 1174-1188, doi:10.1016/j.neuron.2016.05.005 (2016).

31 Carbon, S. et al. AmiGO: online access to ontology and annotation data. Bioinformatics 25, 288-289, doi:10.1093/bioinformatics/btn615 (2009).

32 Reilly, S. K. et al. Evolutionary genomics. Evolutionary changes in promoter and enhancer activity during human corticogenesis. Science 347, 1155-1159, doi:10.1126/science.1260943 (2015).

33 Visel, A., Minovitsky, S., Dubchak, I. \& Pennacchio, L. A. VISTA Enhancer Browser--a database of tissue-specific human enhancers. Nucleic Acids Res 35, D88-92, doi:10.1093/nar/gk1822 (2007).

34 Bulik-Sullivan, B. K. et al. LD Score regression distinguishes confounding from polygenicity in genome-wide association studies. Nat Genet 47, 291-295, doi:10.1038/ng.3211 (2015).

35 Finucane, H. K. et al. Partitioning heritability by functional annotation using genome-wide association summary statistics. Nat Genet 47, 1228-1235, doi:10.1038/ng.3404 (2015).

36 Demontis, D. et al. Discovery of the first genome-wide significant risk loci for attention deficit/hyperactivity disorder. Nat Genet 51, 63-75, doi:10.1038/s41588-018-0269-7 (2019).

37 Grove, J. et al. Identification of common genetic risk variants for autism spectrum disorder. Nat Genet 51, 431-444, doi:10.1038/s41588-019-0344-8 (2019).

38 Stahl, E. A. et al. Genome-wide association study identifies 30 loci associated with bipolar disorder. Nat Genet 51, 793-803, doi:10.1038/s41588-019-0397-8 (2019).

39 Savage, J. E. et al. Genome-wide association meta-analysis in 269,867 individuals identifies new genetic and functional links to intelligence. Nat Genet 50, 912-919, doi:10.1038/s41588-018-0152-6 (2018).

40 Howard, D. M. et al. Genome-wide meta-analysis of depression identifies 102 independent variants and highlights the importance of the prefrontal brain regions. Nat Neurosci 22, 343-352, doi:10.1038/s41593-018-0326-7 (2019).

41 Pardinas, A. F. et al. Common schizophrenia alleles are enriched in mutation-intolerant genes and in regions under strong background selection. Nat Genet 50, 381-389, doi:10.1038/s41588-018-0059-2 (2018).

42 Song, M. et al. Mapping cis-regulatory chromatin contacts in neural cells links neuropsychiatric disorder risk variants to target genes. Nat Genet 51, 1252-1262, doi:10.1038/s41588-019-0472-1 (2019).

43 Fang, R. et al. Mapping of long-range chromatin interactions by proximity ligation-assisted ChIP-seq. Cell Res 26, 1345-1348, doi:10.1038/cr.2016.137 (2016). 
$107044 \quad$ Hnisz, D. et al. Super-enhancers in the control of cell identity and disease. Cell 155, 9341071947 , doi:10.1016/j.cell.2013.09.053 (2013).

107245 Xie, S., Duan, J., Li, B., Zhou, P. \& Hon, G. C. Multiplexed Engineering and Analysis of 1073 1074 1075 Combinatorial Enhancer Activity in Single Cells. Mol Cell 66, 285-299 e285, doi:10.1016/j.molcel.2017.03.007 (2017). Methods 14, 297-301, doi:10.1038/nmeth.4177 (2017). 


\section{Figure 1}

a

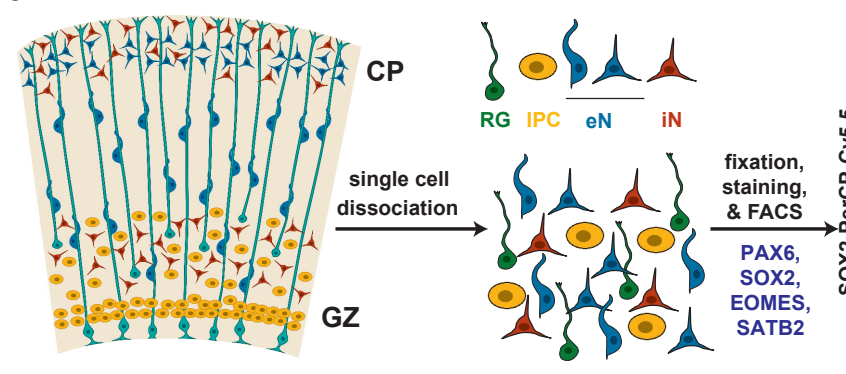

b

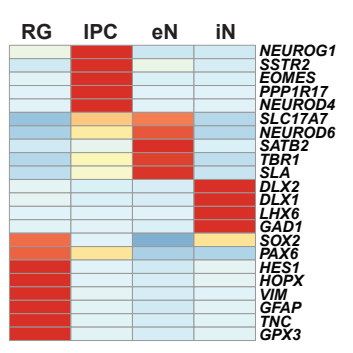

C

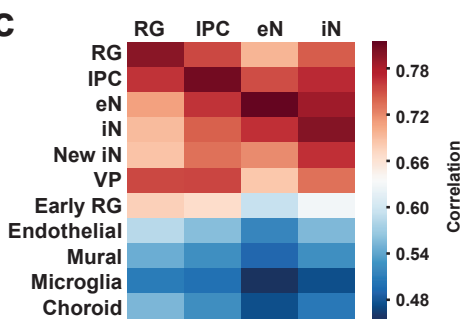

e

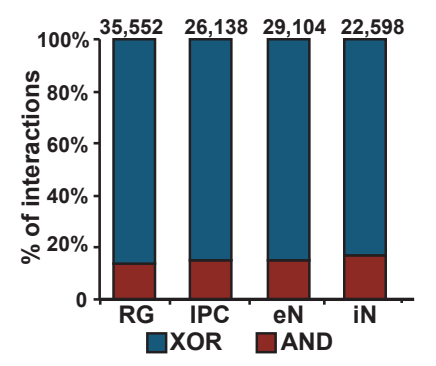

d

$\mathbf{f}$
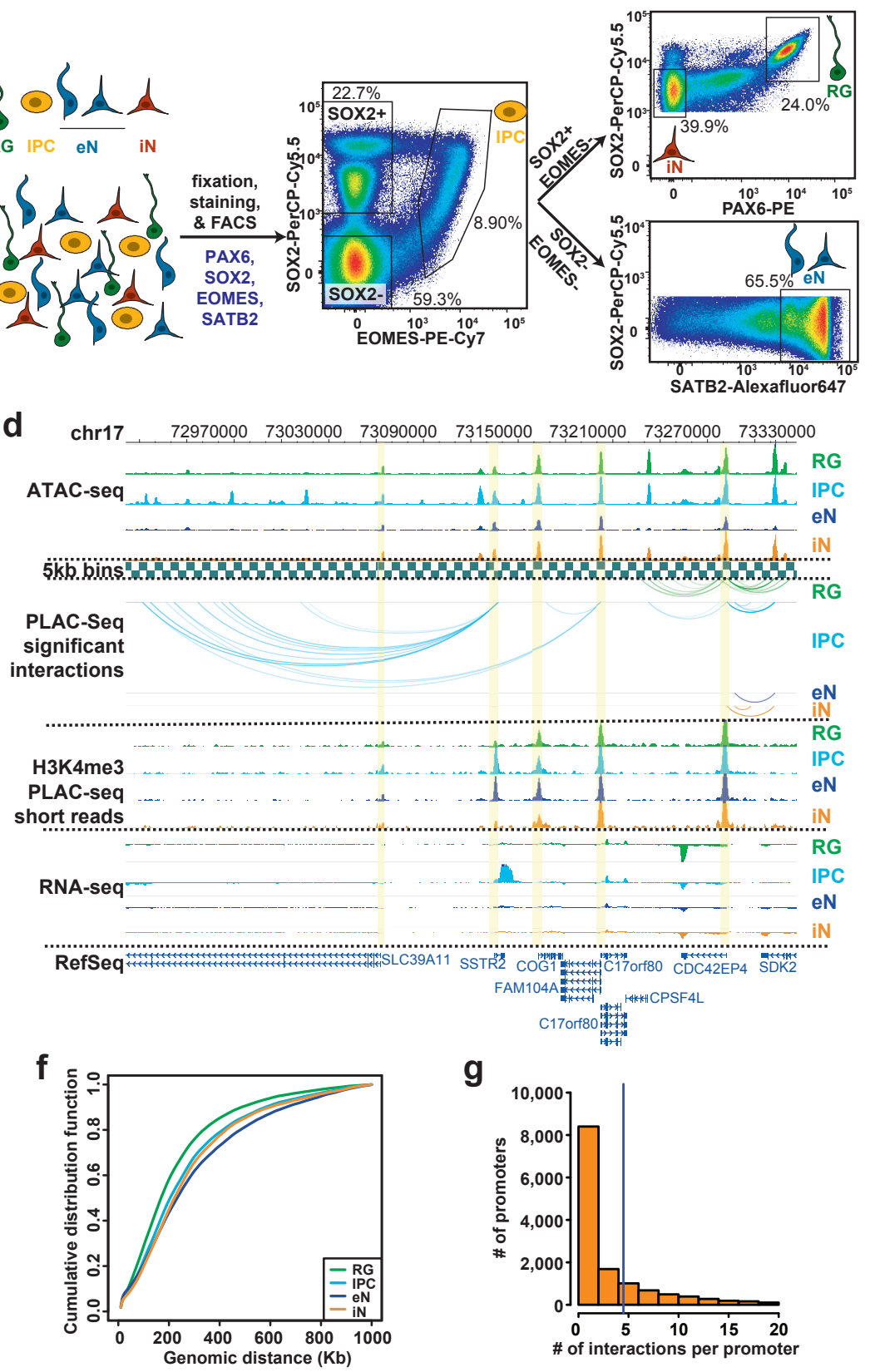

Figure 1. Experimental design and general features of the 3D epigenomic landscape during corticogenesis.

(a) Schematic of the sorting strategy. Within the dorsal cortex, the germinal zone (GZ) is populated by radial glia (RG), which extend fibers towards the cortical plate (CP). These RG divide asymmetrically to produce intermediate progenitor cells (IPCs), which differentiate into excitatory neurons (eNs) that migrate along RG fibers towards the CP. At the same time, interneurons (iNs) can be found in both the GZ and CP. Microdissected GZ and CP samples were dissociated into single cells before being fixed, stained with antibodies for EOMES, SOX2, PAX6, and SATB2, and sorted using FACS. (b) Heatmap showing expression of key marker genes for RG, IPCs, eNs, and iNs. (c) Heatmap showing correlations between gene expression profiles for sorted cell populations and aggregate gene expression profiles from scRNA-seq datasets in the developing cortex. Cell types include newborn iNs from the medial ganglionic eminence (MGE), ventral progenitors including RG and IPCs from the MGE, microglia, and choroid plexus cells. (d) WashU Epigenome Browser snapshot of a $360 \mathrm{~kb}$ region (chr17:72,970,0000-73,330,000) showing IPC-specific chromatin interactions linked to SSTR2 expression in IPCs. (e) Bar graph showing counts of MAPS interactions, with proportions of XOR (blue, only one interacting bin contains $\mathrm{H} 3 \mathrm{~K} 4 \mathrm{me} 3$ peaks) and AND (red, both interacting bins contain $\mathrm{H} 3 \mathrm{~K} 4 \mathrm{me} 3$ peaks) interactions displayed for each cell type. (f) Cumulative distribution function (CDF) plots showing interaction distances for each cell type. (g) Histogram showing the numbers of MAPS interactions at each promoter for each cell type. 


\section{Figure 2}

a
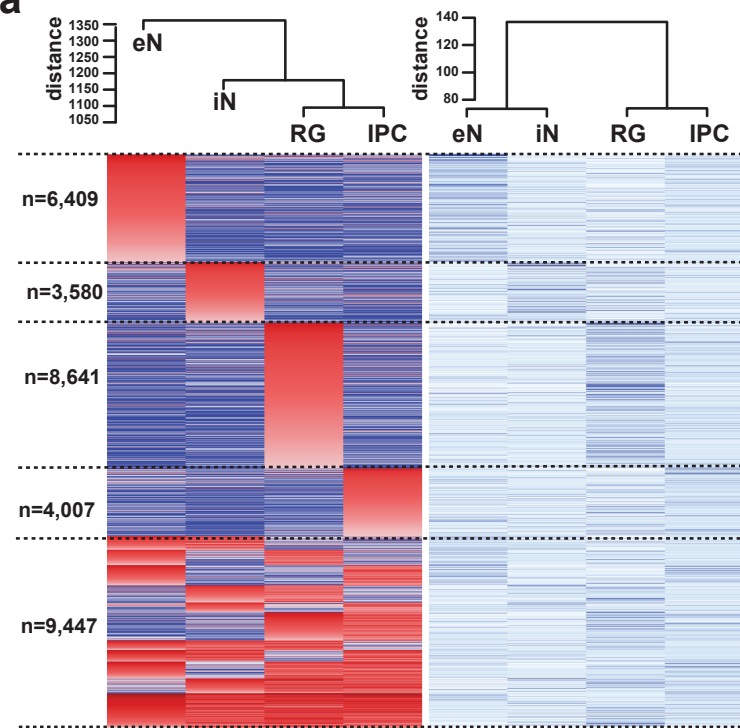

interaction $\left(-\log _{10} \mathrm{FDR}\right)$

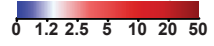

b

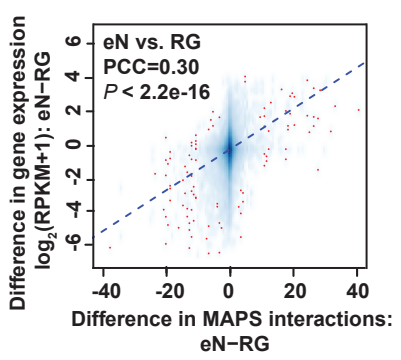

C

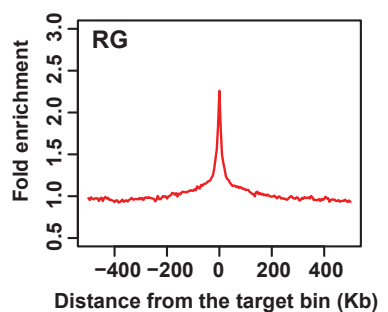

d

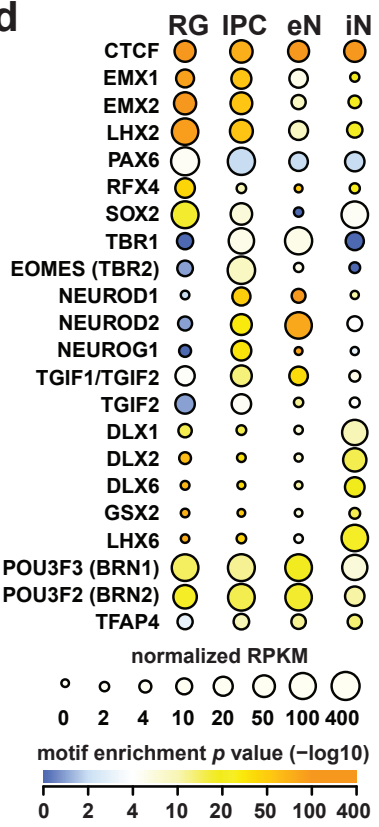

Figure 2. H3K4me3-mediated chromatin interactions contribute to cell type-specific gene regulation.

(a) Heatmaps displaying interaction scores (left) and gene expression (right) for unique XOR interactions grouped according to their cell type specificity. Hierarchical clustering dendrograms for each heatmap are also shown (top).

(b) Scatterplot showing positive correlation between the difference in the number of MAPS interactions at each promoter and the difference in expression of the corresponding genes between RG and eNs (Pearson productmoment correlation coefficient, two-sided t-test, $P<2.2 \times 10^{-16}$ ). The fitted trendline based on linear regression is also shown. (c) Fold enrichment of open chromatin regions over distance-matched background regions in $1 \mathrm{Mb}$ windows around distal interacting regions for MAPS interactions in RG. (d) Enrichment of TF motifs at open chromatin regions in cell type-specific interacting distal regions for each cell type. The color of each dot represents the degree of enrichment $\left(-\log _{10} P\right.$-value $)$ for each TF motif, and the size of each dot represents the gene expression of the corresponding TF. 
bioRxiv preprint doi: https://doi.org/10.1101/2020.02.24.963652; this version posted February 25, 2020. The copyright holder for this preprint Figure 3 (which was not certified by peer review) is the author/funder. All rights reserved. No reuse allowed without permission.

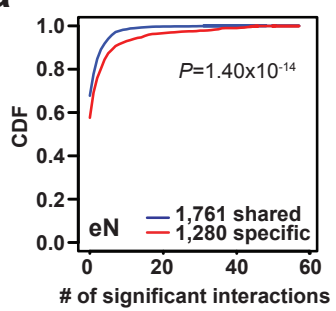

C

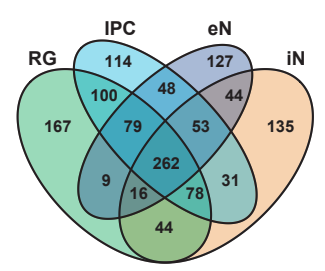

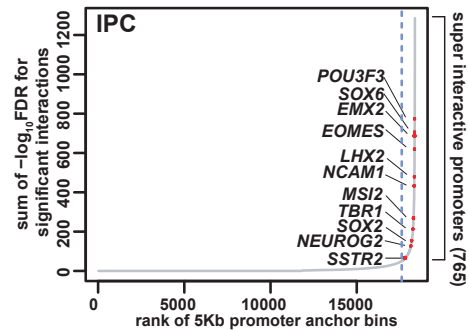

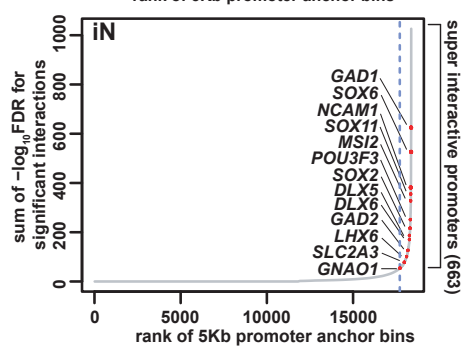

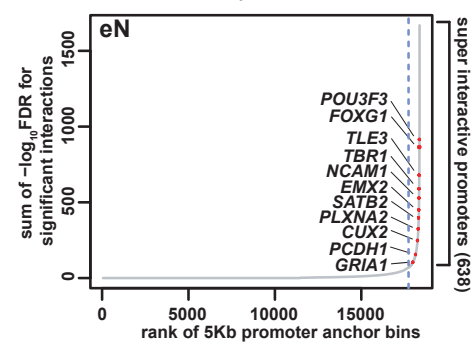
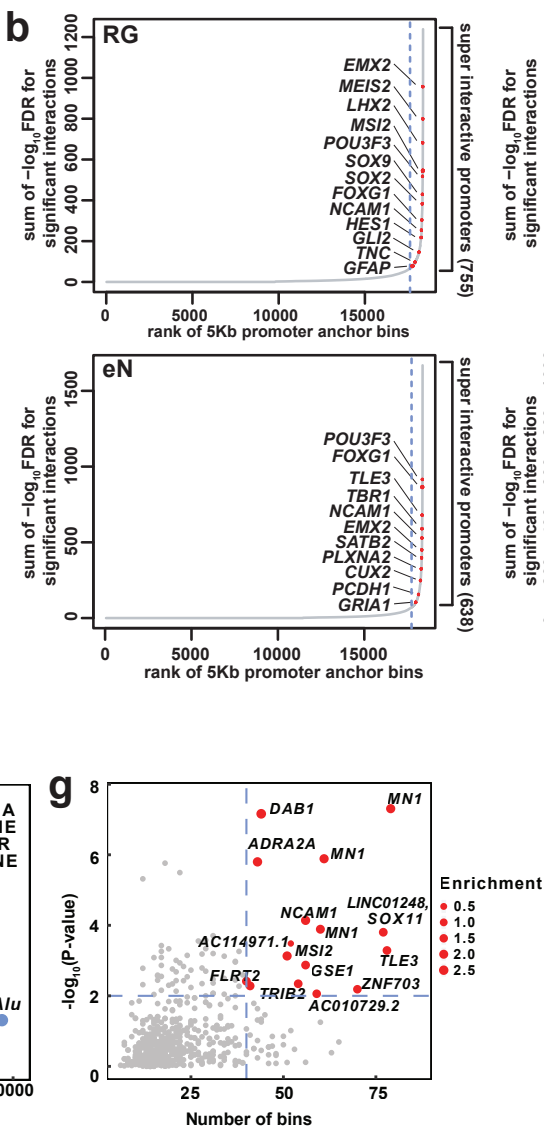

h

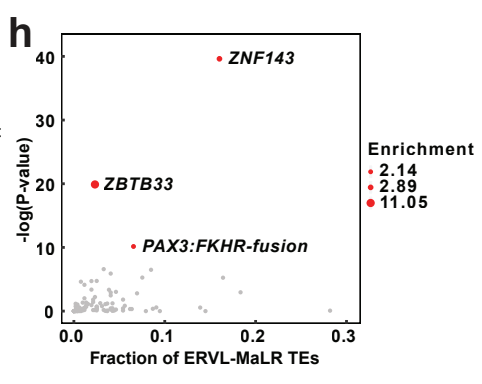

f

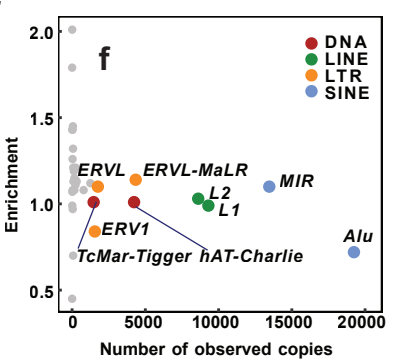

j

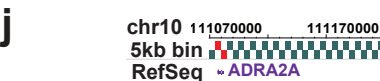

5kb bin (2) ERVL-MaLR masker bin identifie

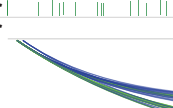

PLAC-seq
eN

RG

IPC

iN d gene expression ranking cochran-Armitage 1st 2nd 3rd 4th Trend Test $(P$ value $)$

$\begin{array}{lllll}\text { RG } \bigcirc & \bigcirc & \circ & 0 & 7.55 \times 10^{-8}\end{array}$

IPC $\bigcirc \bigcirc \bigcirc \bigcirc \quad 0 \quad 1.85 \times 10^{-8}$

eN $\bigcirc \quad O \quad \circ \quad 0 \quad 4.91 \times 10^{-13}$

iN $\bigcirc \circ \circ \circ \quad 0 \quad 3.21 \times 10^{-9}$

fold enrichment over lowest

gene expression (4th) Chi-square test $P$ value

$00 \bigcirc \bigcirc \bigcirc$

$\begin{array}{lllll}1.0 & 1.5 & 2.0 & 2.5 & 3.0\end{array}$

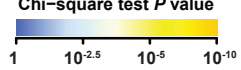

e Cell-type-specific gene enrichment
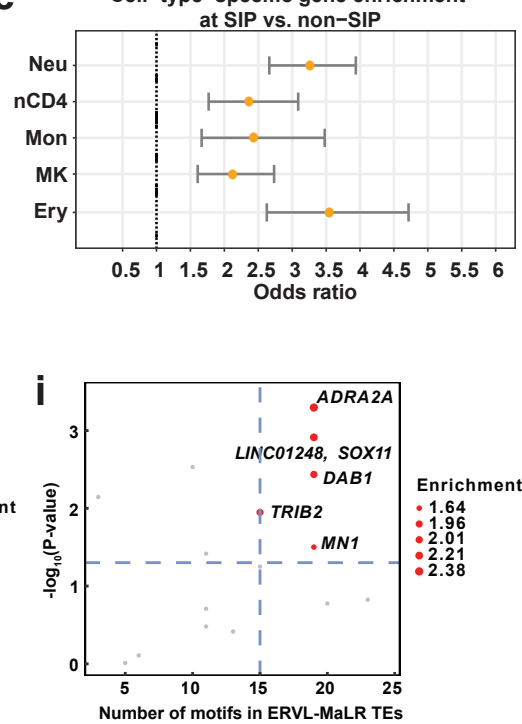

k

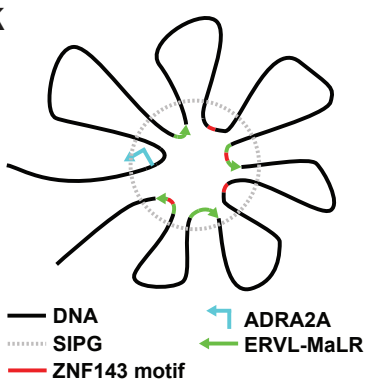

Figure 3. Super interactive promoters are enriched for lineage-specific genes.

(a) CDF plots showing the numbers of MAPS interactions for shared versus cell type-specific genes in eNs (two sample t-test, two-sided, $P=1.40 \times 10^{-14}$ ). (b) Plots showing the ranked cumulative interaction scores for 3D anchor bins in each cell type, defined as the sum of the $-\log _{10}$ FDR for MAPS interactions coincident to each bin. Super interactive promoters (SIPs) are defined as promoters located to the right of the knee of each curve (dashed lines). Example SIPs, including those for lineage-specific genes, are highlighted for each cell type. (c) Venn diagram displaying the cell type-specificity of SIPs in RG, IPCs, eNs, and iNs. (d) The number of genes called as SIPs was divided by the total number of SIPs and non -SIPs for genes with the $1^{\text {st }}, 2^{\text {nd }}, 3^{\text {rd }}$, and $4^{\text {th }}$ highest expression among all four cell types. The fold enrichment was calculated relative to the group with the $4^{\text {th }}$ highest expression for each cell type. (e) Forrest plot showing that SIPs called in hematopoietic cells are also enriched for cell type-specific over shared genes. 95\% confidence intervals are displayed. (f) Scatterplot showing both the enrichment and the number of observed copies of TE families in SIPGs for eNs. TE families occupying more than $1 \%$ of the genome are colored. (g) Scatterplot showing the enrichment of ERVL-MaLR TEs in SIPGs for eNs (hypergeometric P-value, see methods). SIPGs with 40 or more distal interacting bins and $P<0.01$ are highlighted. (h) Scatterplot showing the enrichment of TF motifs at ERVL-MaLR TEs in SIPGs highlighted in (g). Only TF motifs with length > 12 bp are shown. (i) Scatterplot showing the enrichment of ZNF143 motifs at ERVL-MaLR TEs in SIPGs highlighted in (g) (Poisson distribution, see methods). ZNF143 motifs occurrences were detected using FIMO using a threshold of $P=$ 0.0001. (j) WashU Epigenome Browser snapshot of the ADRA2A SIPG. MAPS interactions targeting the 12 distal interacting bins containing ERVL-MaLR-derived ZNF143 motifs are highlighted. (k) Potential mechanism for the contributions of TEs towards SIP formation. 


\section{Figure 4}

a

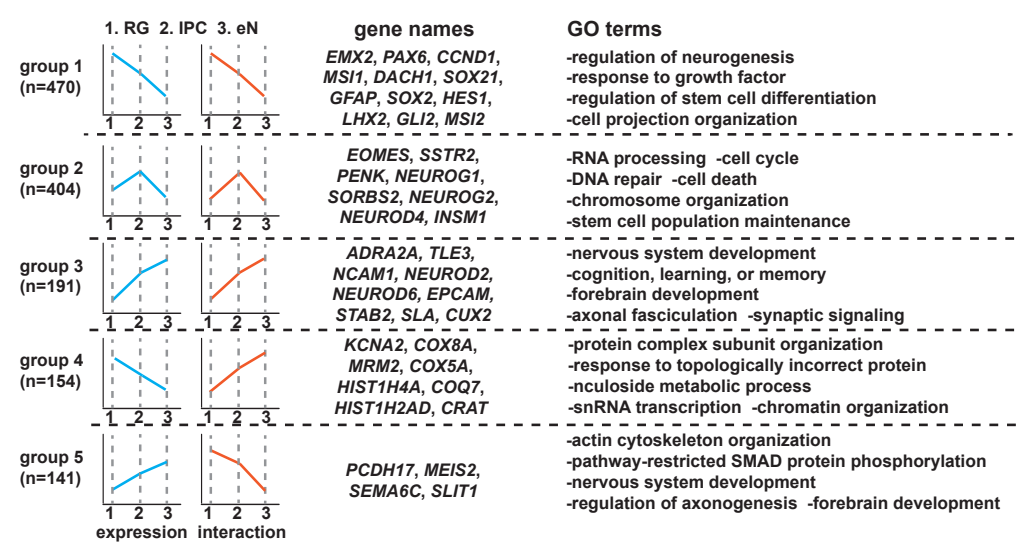

C

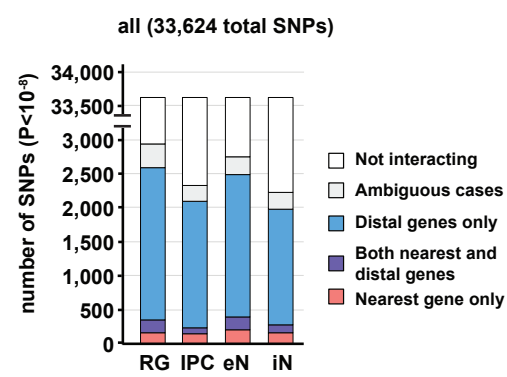

b

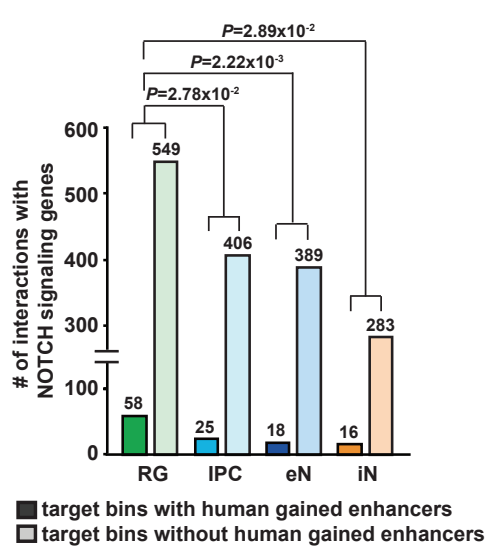

d

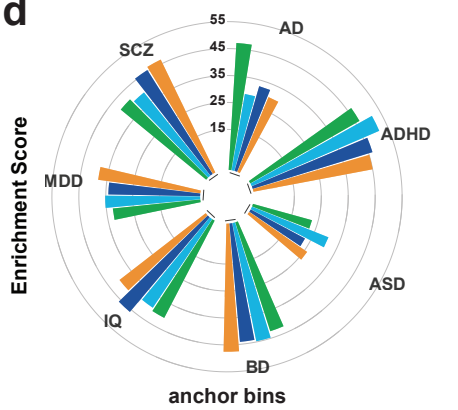

e

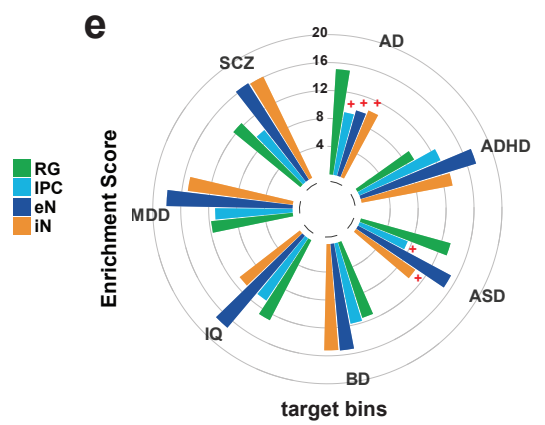

Figure 4. Investigating developmental trajectories during corticogenesis and partitioning heritability for complex neuropsychiatric disorders and traits.

(a) Gene groups identified based on their changes in expression and chromatin interactivity along the transition from RG to eNs. Group 1 represents stem cell genes with decreasing expression and chromatin interactivity from RG to eNs. Group 2 represents IPC-specific genes with the highest expression and chromatin interactivity at the IPC stage. Group 3 represents genes with increasing expression and chromatin interactivity from RG to eNs. Groups 4 and 5 are characterized by anti-correlated expression and chromatin interactivity and may represent latesilenced and early-silenced genes, respectively. Representative genes and GO terms are shown for each group. (b) Bar graph showing the numbers of MAPS interactions at Notch signaling genes targeting bins with and without human gained enhancers in each cell type (Chi-square test). (c) Bar graph showing the numbers of unique GWAS SNPs $\left(P<10^{-8}\right)$ interacting with their nearest gene only, with both their nearest and distal genes, or with distal genes only for each cell type across all neuropsychiatric traits. (d-e) LDSC enrichment scores for each neuropsychiatric trait and cell type, stratified by 3D anchor and target bins. Results with $P>0.05$ are indicated. 

Figure 5 (which was not certified by peer review) is the author/funder. All rights reserved. No reuse allowed without permission.

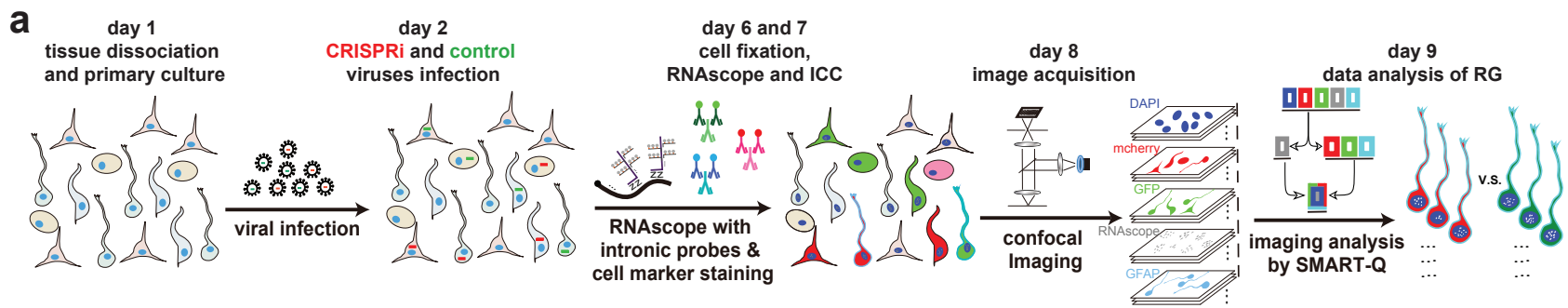

b GPX3

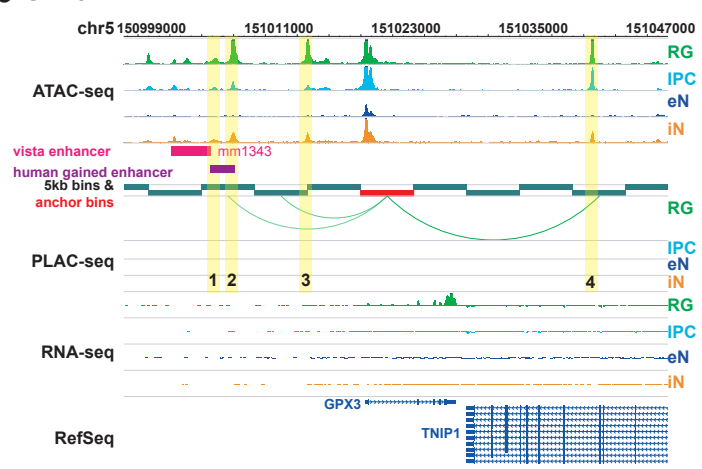

C TNC

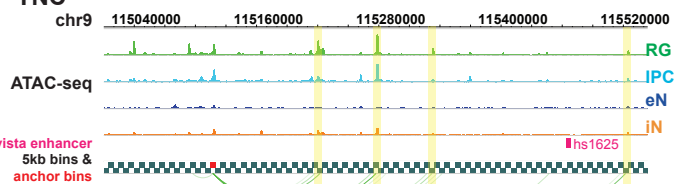

Ins bins \&
anchor bins

PLAC-seq

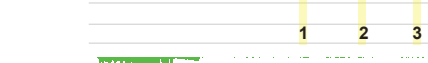

RNA-seq

RefSeq

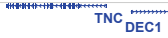

d HES1

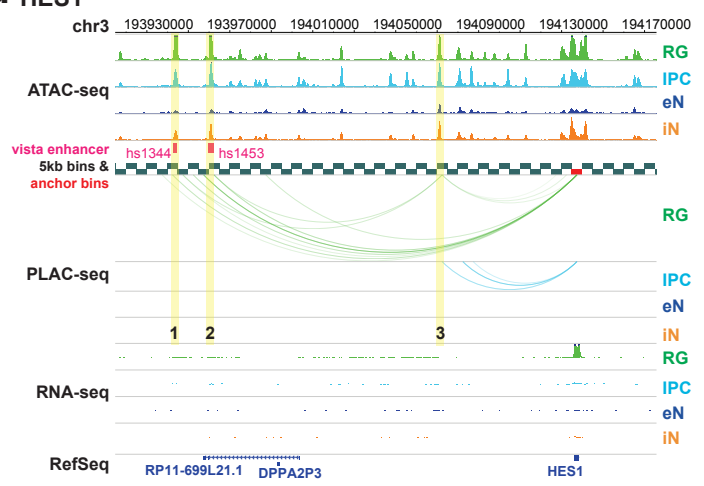

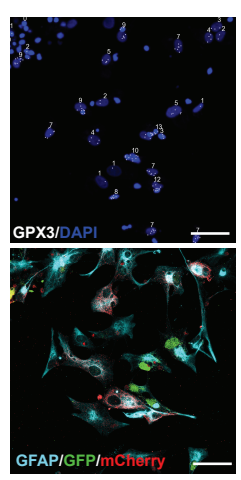
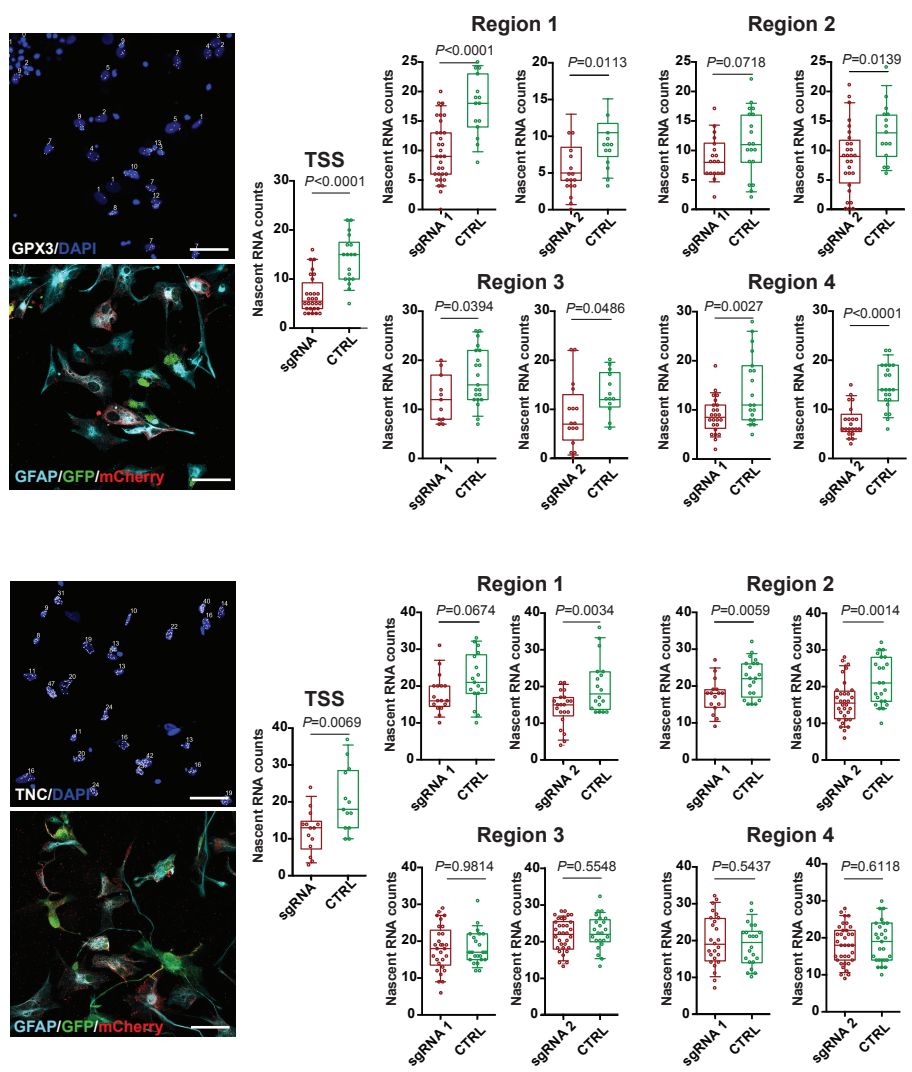

Figure 5. Functional characterization of distal interacting regions using CRISPRview.

(a) CRISPRview workflow. Image analysis was performed using the SMART-Q pipeline. (b-d) Functional characterization of distal interacting regions at the GPX3, TNC, and HES1 loci. For each locus, a WashU Epigenome Browser snapshot shows chromatin interactions bridging the promoters of GPX3,TNC, and HES1 and distal interacting regions containing open chromatin regions (highlighted) which were targeted by sgRNAs for CRISPRi silencing. Representative images show staining for RNAscope probes targeting intronic regions for the genes of interest (white), DAPI (blue), the RG marker GFAP (light blue), mCherry (red), and GFP (green). The scale bar is $50 \mu \mathrm{m}$. Box plots show the results of CRISPRi silencing for each targeted region. The open circles represent single cells, and nascent transcript counts for experimental $(\mathrm{mCherry}+)$ versus control (GFP+) sgRNA-treated RG are represented on the y-axis (Student's t-test, two-tailed). The median, upper and lower quartiles, and $10 \%$ to $90 \%$ range are indicated. 
bioRxiv preprint doi: https://doi.org/10.1101/2020.02.24.963652; this version posted February 25, 2020. The copyright holder for this preprint (which was not certified by peer review) is the author/funder. All rights reserved. No reuse allowed without permission.

\section{Supplementary Figure 1}

a

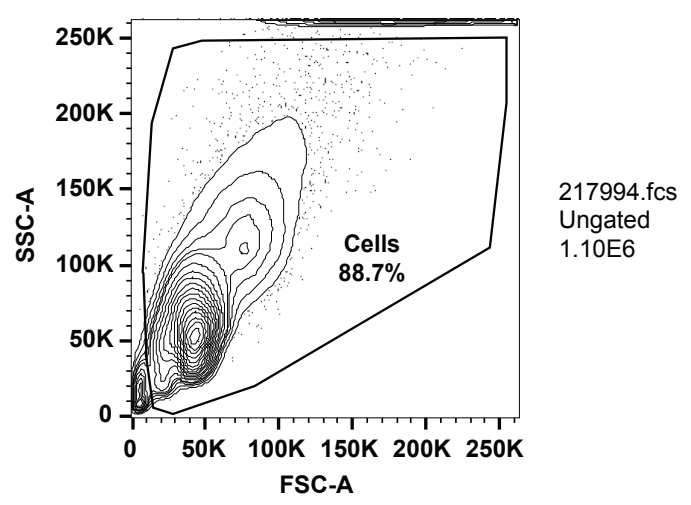

C

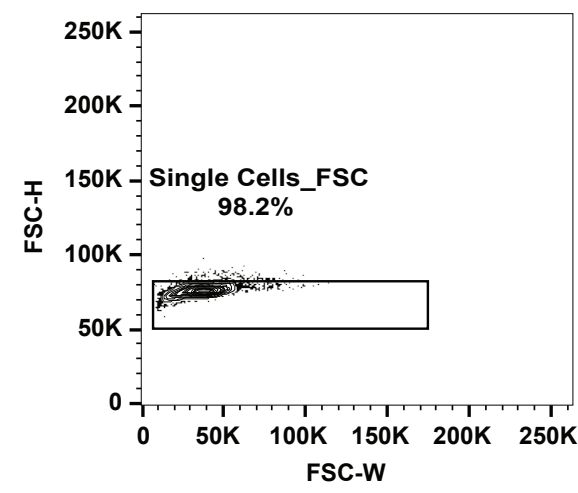

b

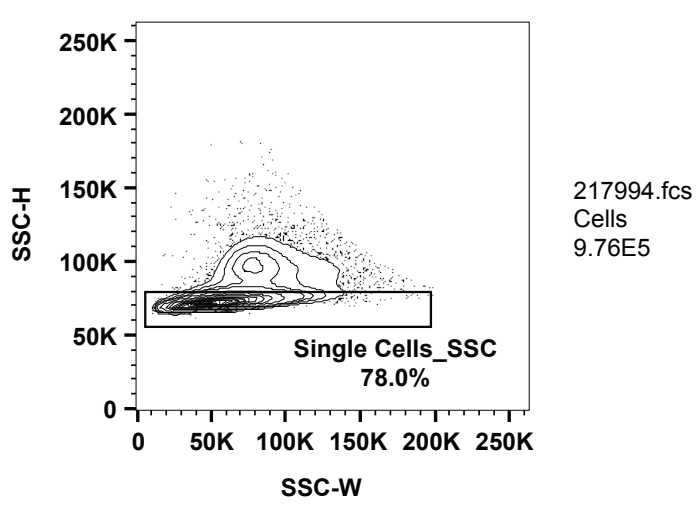

d

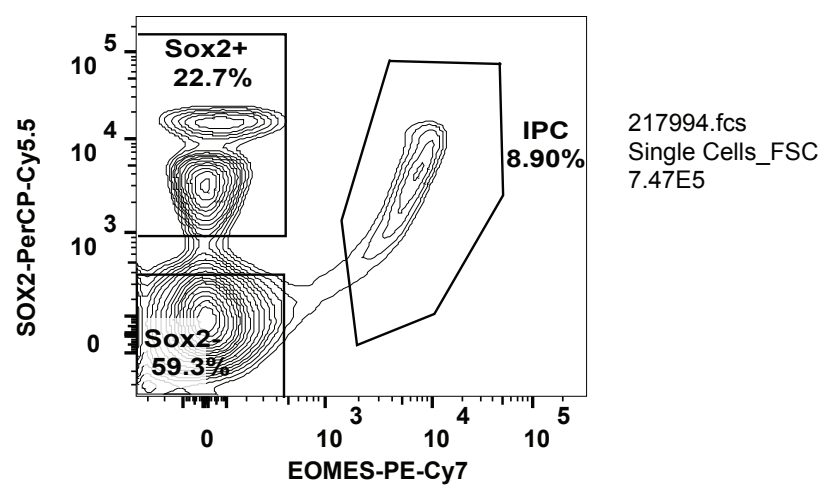

f

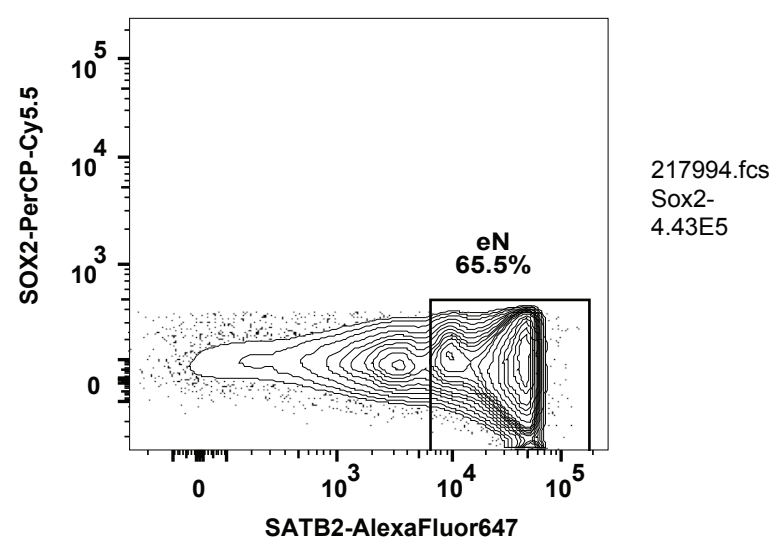

Supplemental Figure 1. Representative contour plots depicting FACS gating strategy.

(a) Cells were separated from debris of various sizes based on the forward scatter area (FSC-A) and side scatter area (SSC-A). Cells were then passed through two singlet gates using the width and height metrics of the (b) side scatter (SSC-H versus SSC-W) and (c) forward scatter (FSC-H versus FSC-W). (d) SOX2+, and SOX2-, and intermediate progenitor (IPC) populations were isolated by gating on EOMES-PE-Cy7 and SOX2-PerCP-Cy5.5 staining. (e) Radial glia (RG) and interneurons (iNs) were isolated as high PAX6/high SOX2 and medium SOX2/low PAX6 populations, respectively. (f) Excitatory neurons (eNs) were isolated from the SOX2- population by gating on SATB2-Alexa Fluor 647 staining. 
bioRxiv preprint doi: https://doi.org/10.1101/2020.02.24.963652; this version posted February 25, 2020. The copyright holder for this preprint SupplementatychFigeupecetified by peer review) is the author/funder. All rights reserved. No reuse allowed without permission.

a

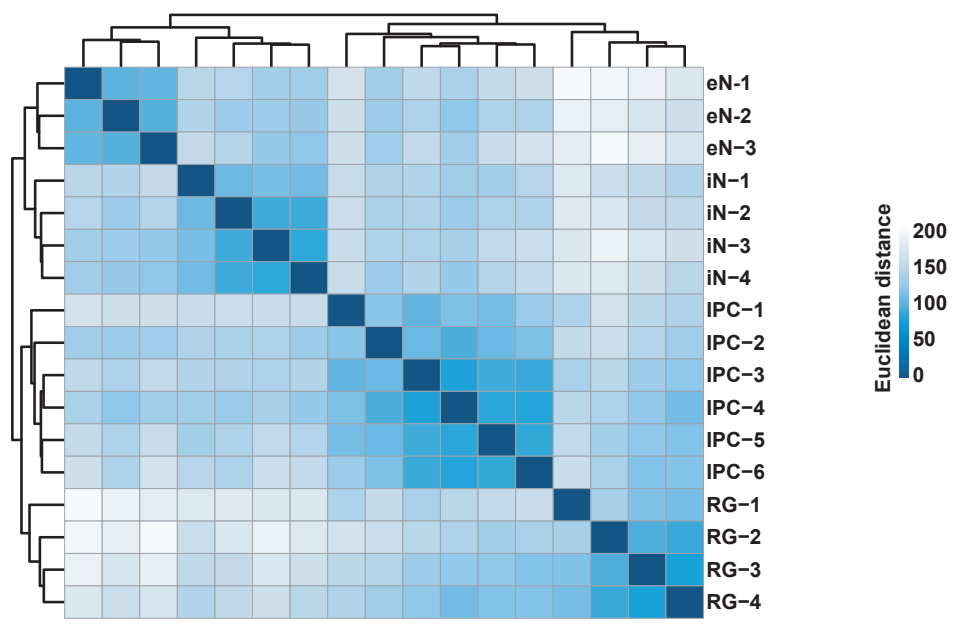

b
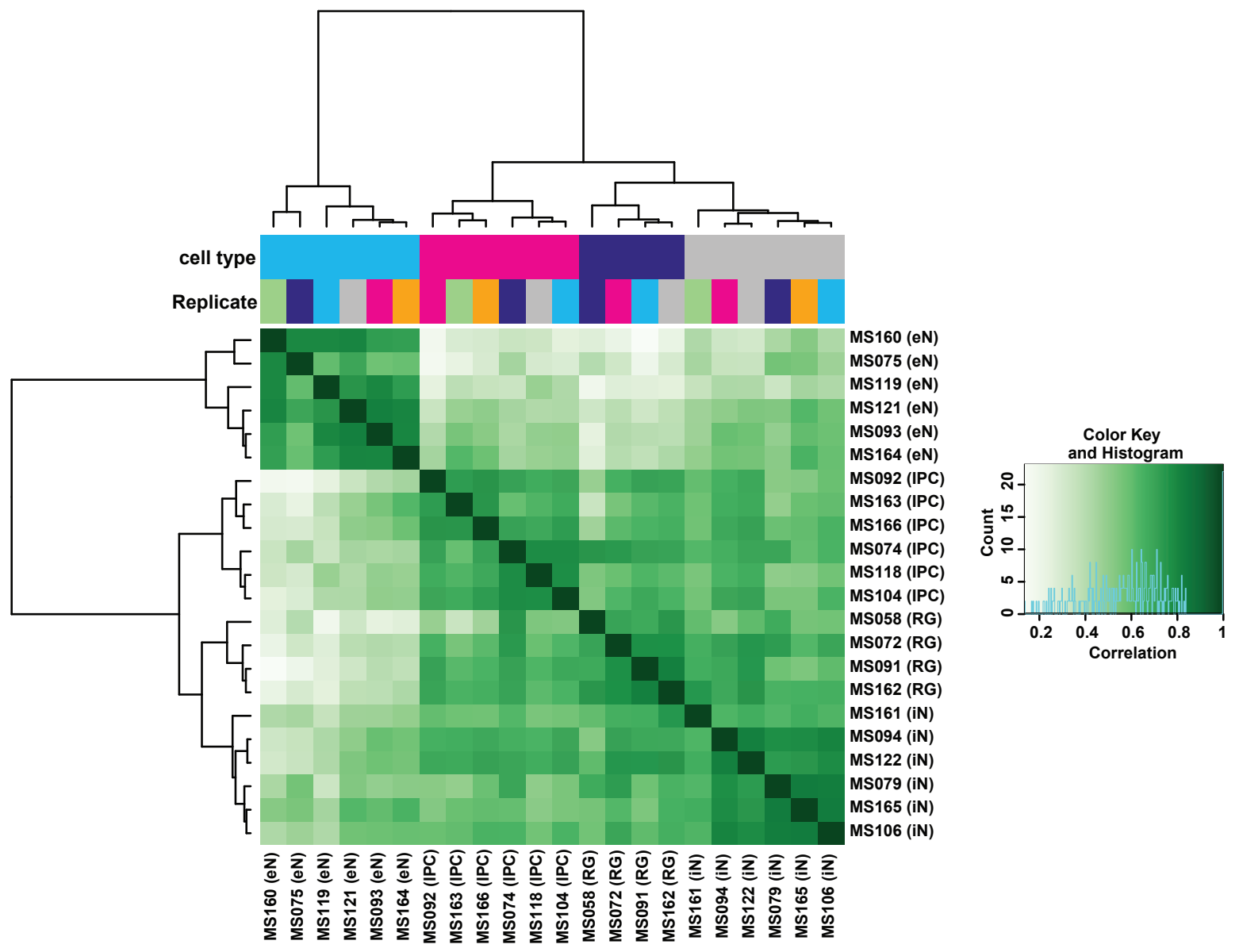

C
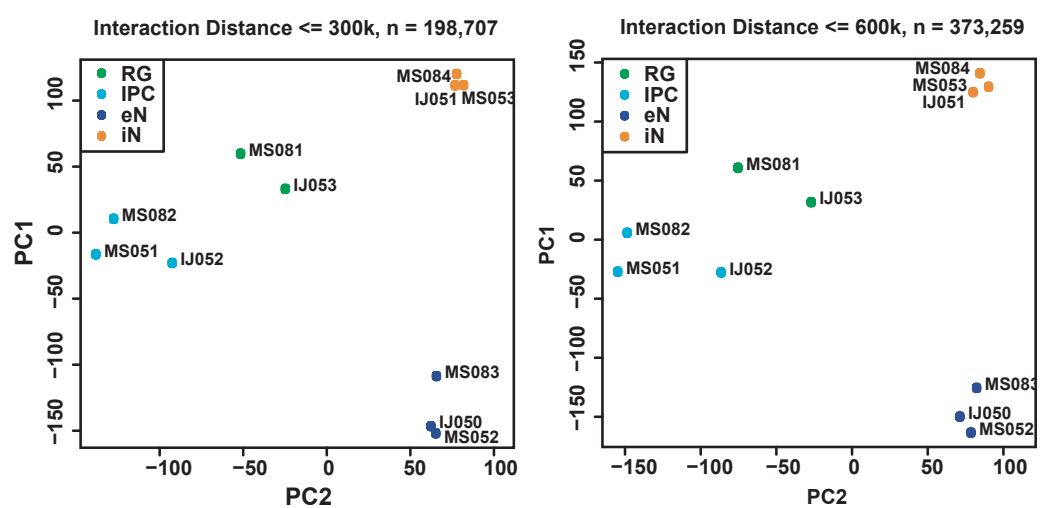

Supplementary Figure 2. Reproducibility between replicates for RNA-seq, ATAC-seq, and PLAC-seq.

(a) RNA-seq replicates were hierarchically clustered according to gene expression sample distances using DESeq2.

(b) Heatmap with pairwise correlations and hierarchical clustering for read densities at the set of unified open chromatin regions for ATAC-seq replicates. (c) Principle component analysis (PCA) was performed based on the normalized contact frequencies across all PLAC-seq replicates (see methods). To assess the robustness of the results, we conducted the analysis separately for bin pairs within 300 and $600 \mathrm{~kb}$ interacting windows. 


\section{Supplementary Figure 3}

a

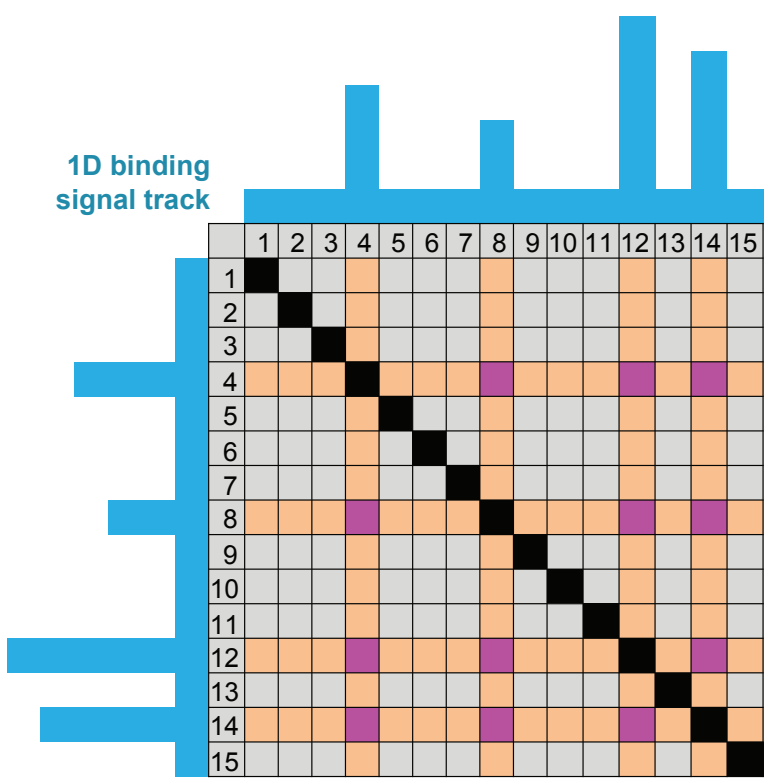

"AND" set: Bin pairs with both ends overlapping protein binding peaks

"XOR" set: Bin pairs with one end overlapping protein binding peaks

"NOT" set: Bin pairs with neither end overlapping protein binding peaks b

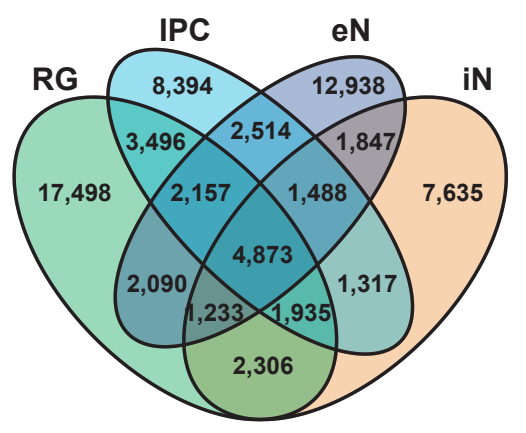

C

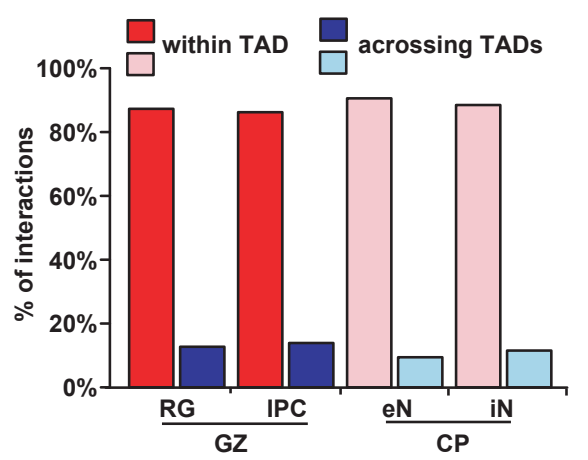

\section{Supplementary Figure 3. Identification of chromatin interactions using MAPS.}

(a) Illustration of AND and XOR sets in a representative PLAC-seq contact matrix. The blue tracks represent 1D H3K4me3 peaks at bin positions 4, 8, 12, and 14. The black cells represent interactions within the same bin. The purple cells represent interactions in the AND set where both of the interacting bins contain 1D H3K4me3 peaks. The orange cells represent interactions in the XOR set where only one of the interacting bins contains 1D H3K4me3 peaks. The grey cells represent interactions where neither of the interacting bins contains 1D H3K4me3 peaks. (b) Venn diagram displaying cell type-specificity of MAPS interactions for each cell type. (c) Proportions of MAPS interactions occurring within and across TADs in GZ and CP tissues for each cell type. 
bioRxiv preprint doi: https://doi.org/10.1101/2020.02.24.963652; this version posted February 25, 2020. The copyright holder for this preprint (which was not certified by peer review) is the author/funder. All rights reserved. No reuse allowed without permission.

\section{Supplementary Figure 4}

a RG terms

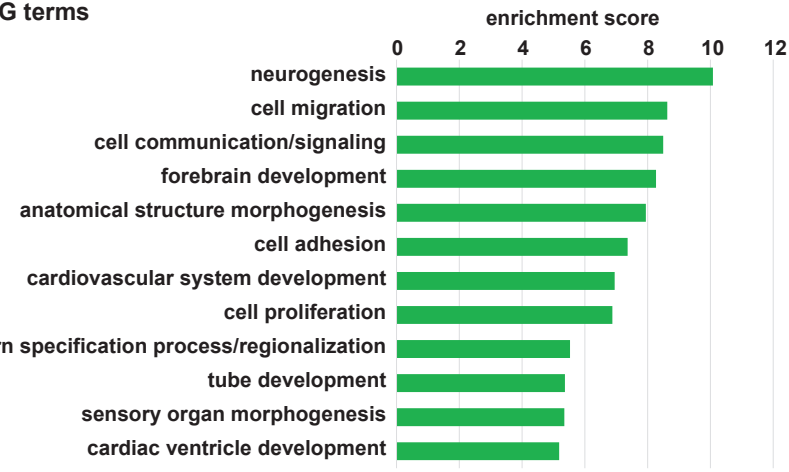

eN terms cardiac ventricle development

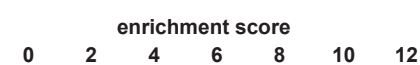

neurogenesis

forebrain development chemical synaptic transmission cell communication/signaling regulation of axonogenesis synapse assembly/organization cognition, learning, or memory cell migration

negative regulation of axonogenesis regulation of ion transport/transporter activity potassium ion transport muscle tissue development

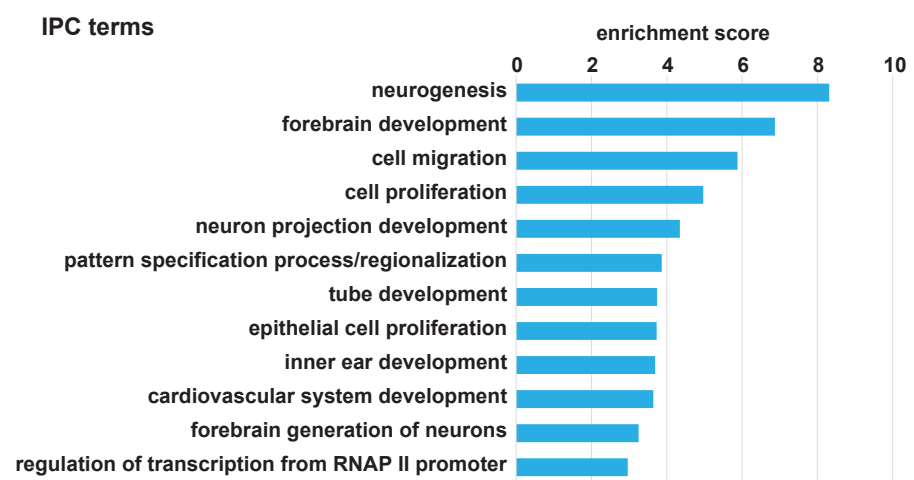

iN terms

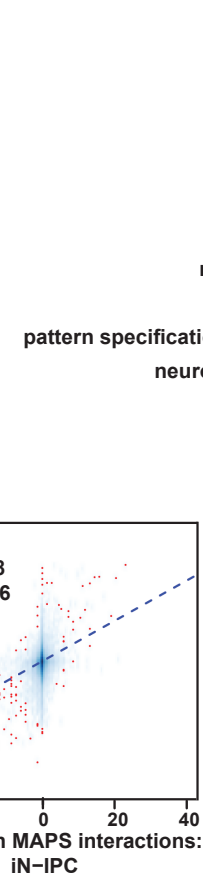

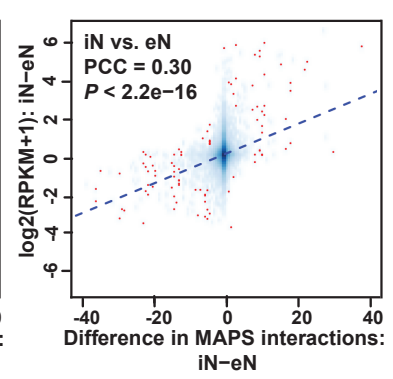

iN-eN enrichment score

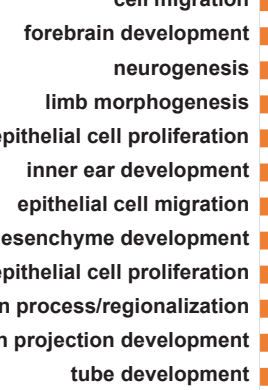

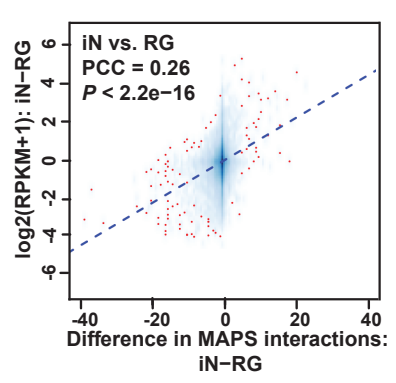

C

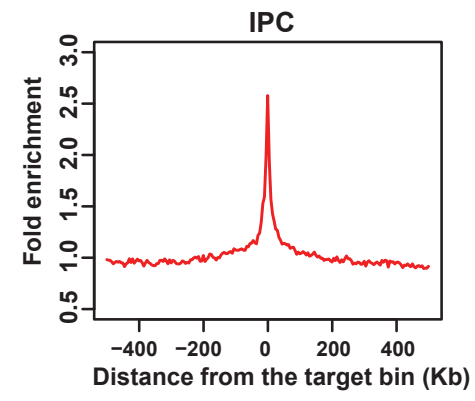

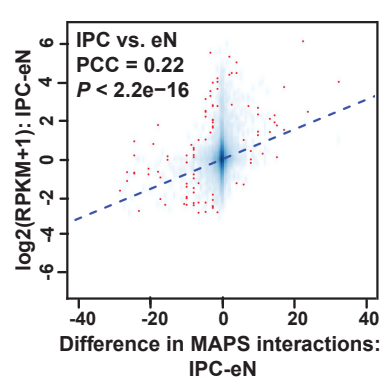

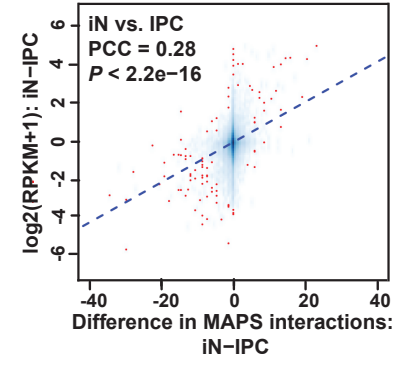

b
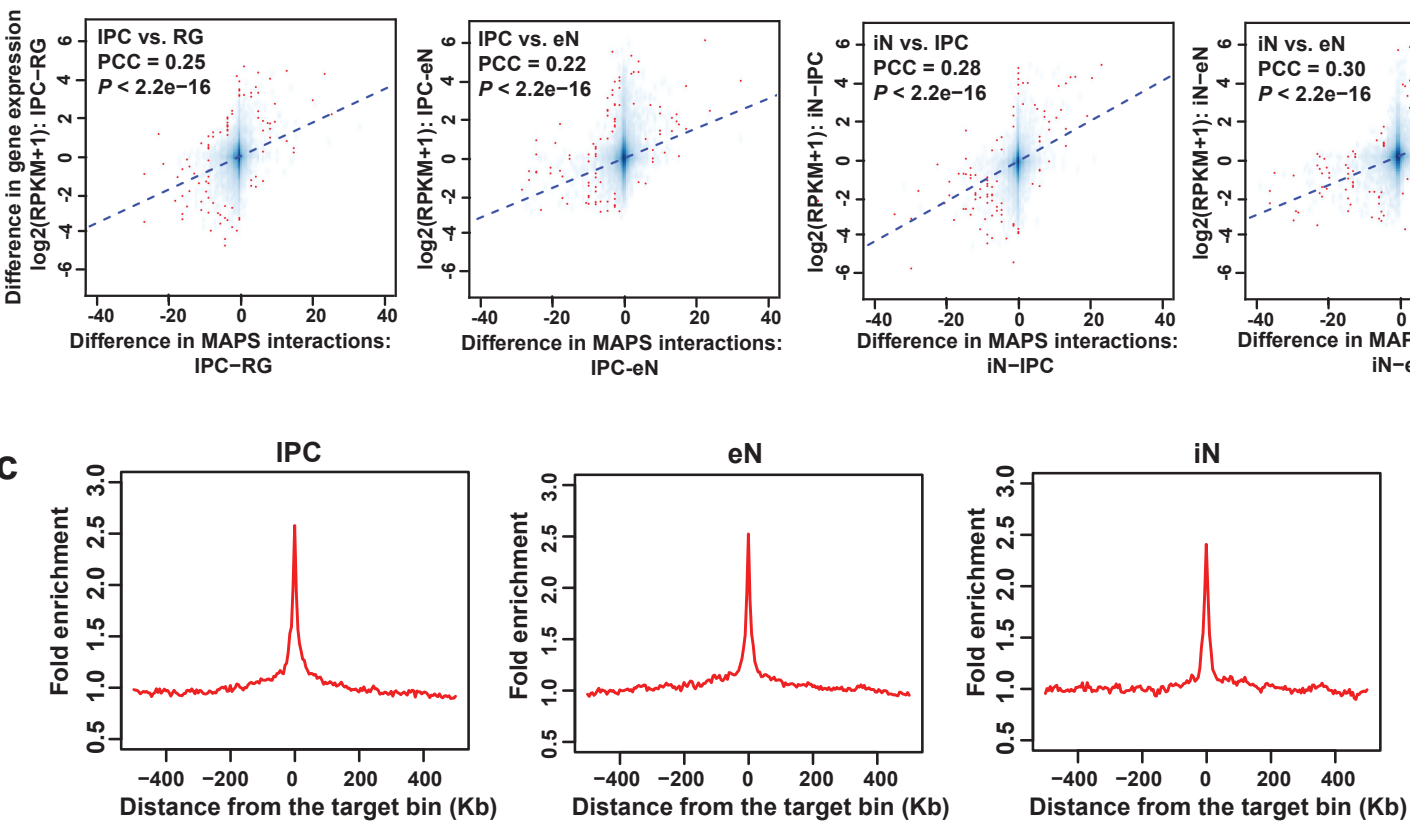

Supplementary Figure 4. Contribution of 3D epigenomic landscapes to gene regulation.

(a) GO enrichment analysis for genes whose promoters participate in cell type-specific interactions. The top annotation clusters from DAVID are reported along with their enrichment scores for each cell type. (b) Scatterplots showing positive correlation between the difference in the number of MAPS interactions at each promoter and the difference in expression of the corresponding genes between all pairs of cell types (Pearson product-moment correlation coefficient, two-sided t-test, $P<2.2 \times 10^{-16}$ for all cell types). Fitted trendlines based on linear regression are also shown. (c) Fold enrichment of open chromatin regions over distance-matched background regions in $1 \mathrm{Mb}$ windows around distal interacting regions for MAPS interactions in IPCs, eNs, and iNs. 


\section{Supplementary Figure 5}

a

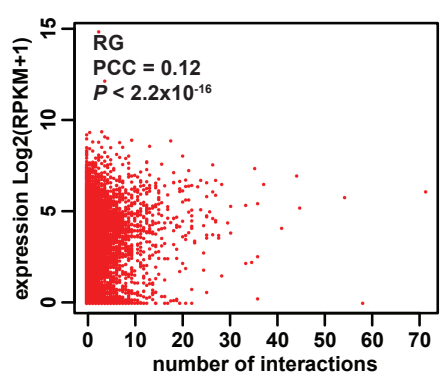

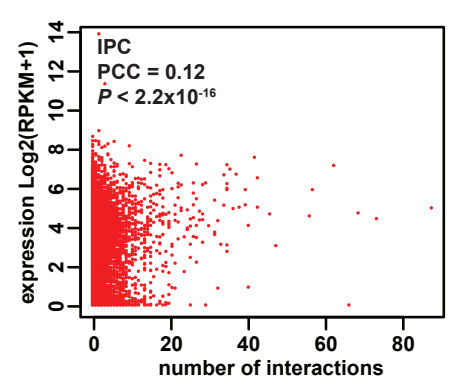
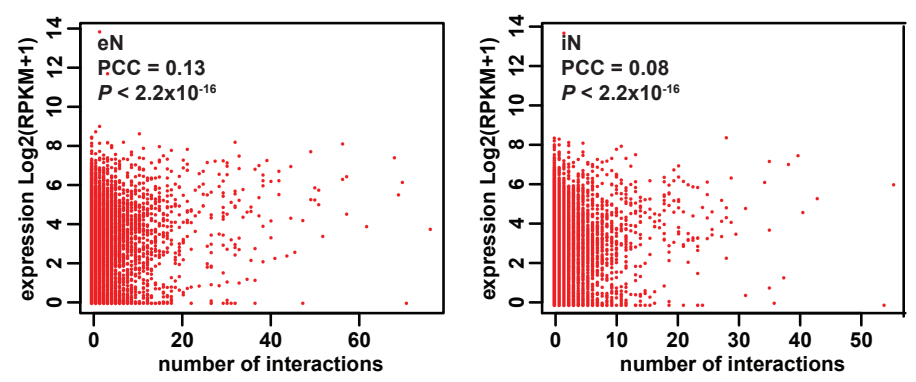

b
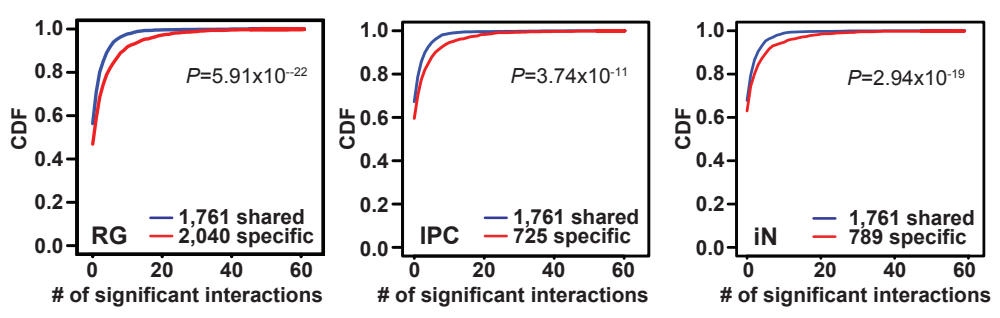

Supplementary Figure 5. Correlations between chromatin interactions and gene expression for cell-type specific and shared genes.

(a) Scatterplots showing the correlation between numbers of MAPS interactions and gene expression at promoters in each cell type. (b) Cumulative distribution function (CDF) plots showing the numbers of MAPS interactions for shared versus cell type-specific genes in RG, IPCs, and iNs (two sample t-test, two-sided, $P=5.91 \times 10^{-22}, 3.74 \times 10^{-11}$, and $2.94 \times 10^{-19}$ for RG, IPCs, and iNs, respectively). 
bioRxiv preprint doi: https://doi.org/10.1101/2020.02.24.963652; this version posted February 25, 2020. The copyright holder for this preprint

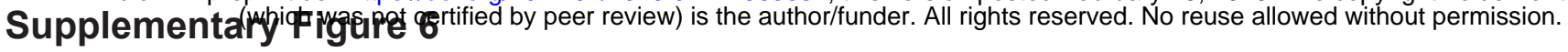
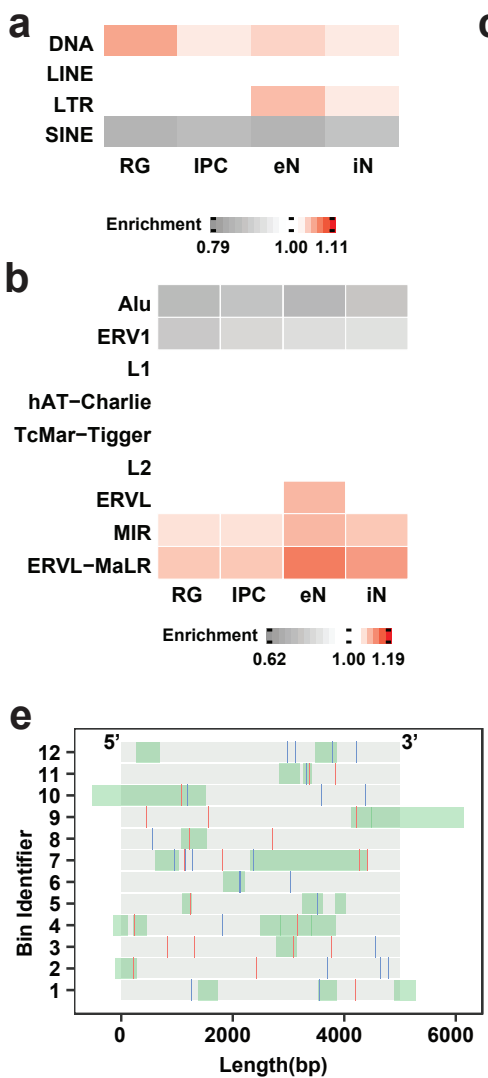

ERVL-MaLR ZZNF143 motif-
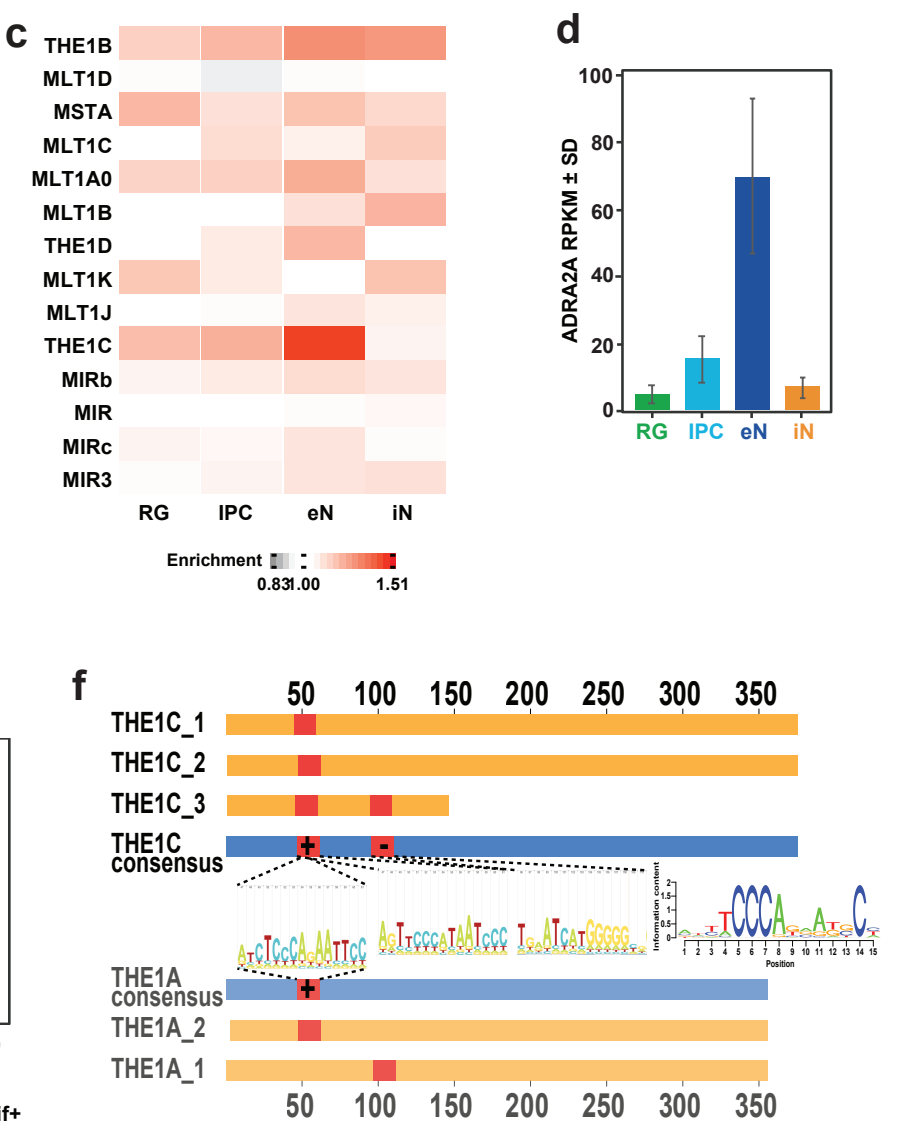

Supplementary Figure 6. Specific families of transposable elements are implicated in SIP formation.

(a-c) Enrichment of TEs in SIPGs at the class (a), family (b), and subfamily (c) levels for each cell type. Only families occupying more than $1 \%$ of the genome are shown in (b). Only subfamilies from the MIR and ERVL-MaLR families occupying more than $0.1 \%$ of the genome are shown in (c). (d) Bar graph shows elevated ADRA2A gene expression in eNs. (e) Illustration of the 12 distal interacting regions in the ADRA2A SIPG containing at least one ERVL-MaLRderived ZNF143 motif. ZNF143 motifs are indicated and colored by strand. The bin identifier corresponds to the labels in Fig. 3j. (f) Illustration of the conservation of ZNF143 binding motifs in ERVL-MaLR TEs. Blue bars indicate consensus sequences, yellow bars indicate individual copies of ERVL-MaLR TEs in the ADRA2A SIPG, and red bars indicate ZNF143 motifs. The positions of the ZNF143 motifs relative to the ERVL-MaLR TE sequences was determined using FIMO. 


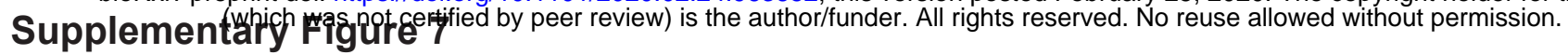

a

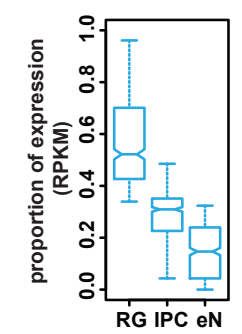

Group 1

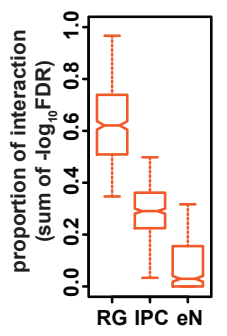

Group 4
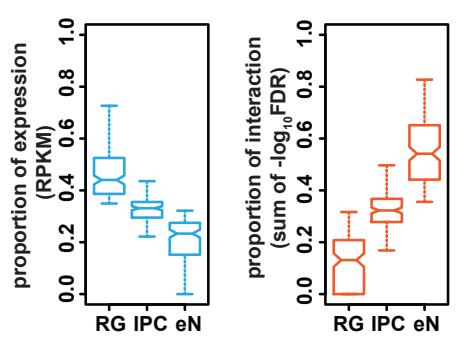

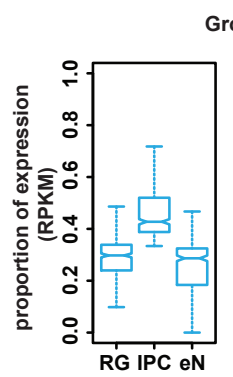

Group 2

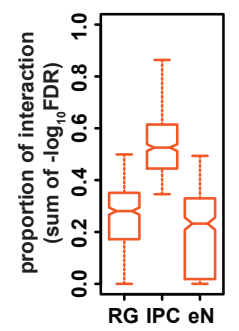

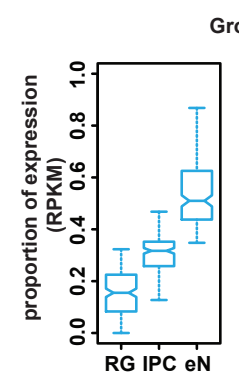

Group 3

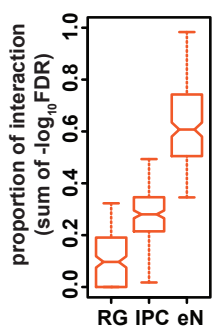

Group 5
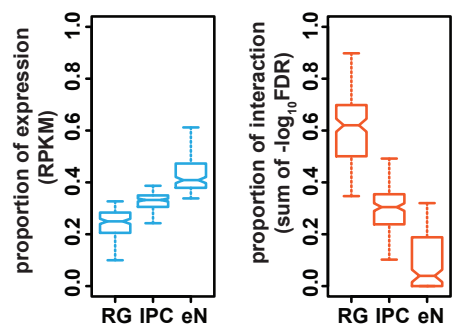

b
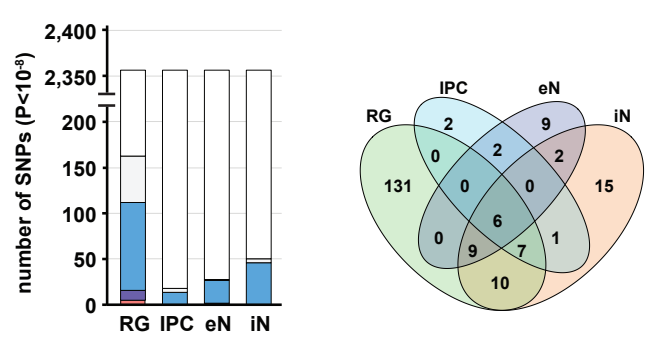

ADHD (317 total SNPs)
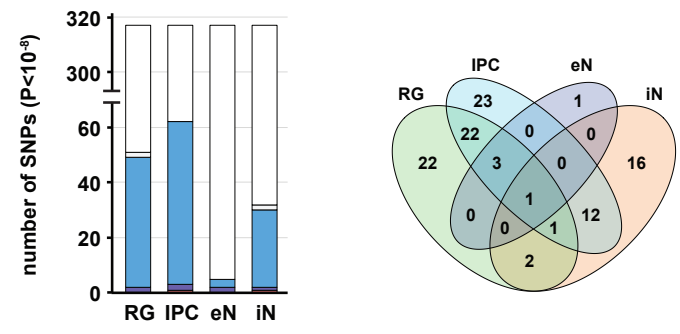

BD (264 total SNPs)
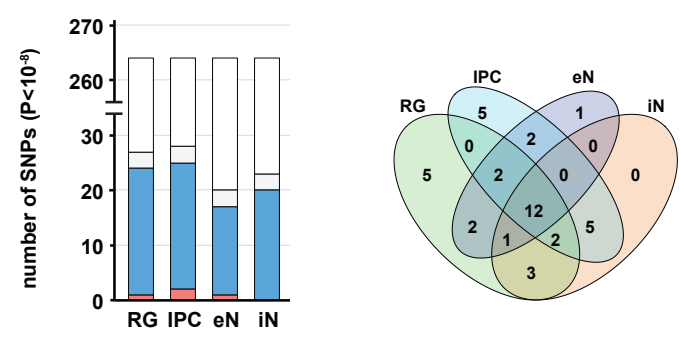

Supplementary Figure 7. Developmental trajectories and annotations for complex neuropsychiatric disorderand trait-associated variants.

(a) Box plots showing the distributions of gene expression and cumulative interaction scores for groups in Fig. 4. The median, upper and lower quartiles, minimum, and maximum are indicated. (b) Bar graphs showing the numbers of GWAS SNPs $\left(P<10^{-8}\right)$ interacting with their nearest gene only, with both their nearest and distal genes, or with distal genes only for each cell type and neuropsychiatric trait. Venn diagrams display the cell type-specificity of all interacting GWAS SNPs for each neuropsychiatric trait.

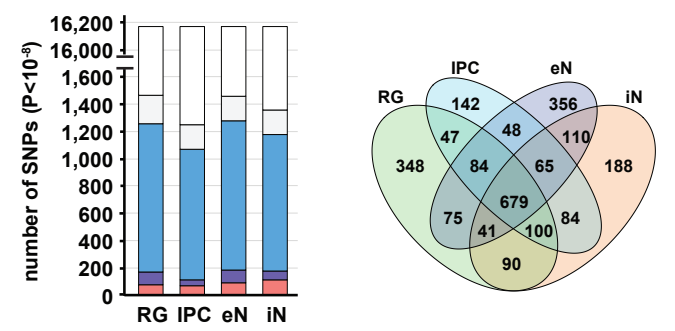

$\square$ Not interacting

$\square$ Ambiguous cases

Distal genes only

$\square$ Both nearest and

$\square$ Nearest gene only 